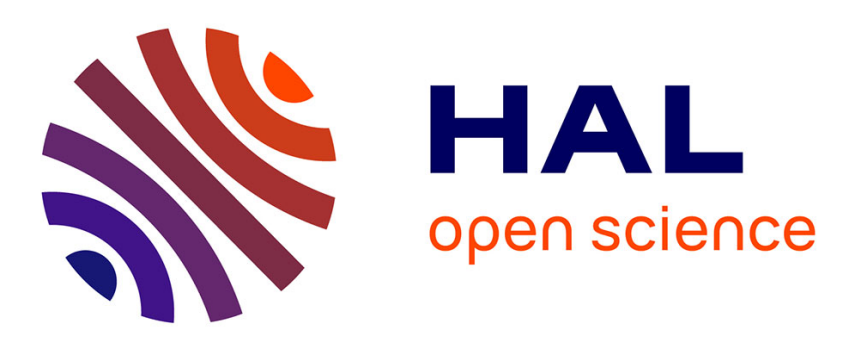

\title{
Moist versus Dry Barotropic Instability in a Shallow-Water Model of the Atmosphere with Moist Convection
}

\author{
Julien Lambaerts, Guillaume Lapeyre, Vladimir Zeitlin
}

\section{To cite this version:}

Julien Lambaerts, Guillaume Lapeyre, Vladimir Zeitlin. Moist versus Dry Barotropic Instability in a Shallow-Water Model of the Atmosphere with Moist Convection. Journal of the Atmospheric Sciences, 2011, 68 (6), pp.1234-1252. 10.1175/2011jas3540.1 . hal-01119312

\author{
HAL Id: hal-01119312 \\ https://hal.science/hal-01119312
}

Submitted on 24 Feb 2015

HAL is a multi-disciplinary open access archive for the deposit and dissemination of scientific research documents, whether they are published or not. The documents may come from teaching and research institutions in France or abroad, or from public or private research centers.
L'archive ouverte pluridisciplinaire HAL, est destinée au dépôt et à la diffusion de documents scientifiques de niveau recherche, publiés ou non, émanant des établissements d'enseignement et de recherche français ou étrangers, des laboratoires publics ou privés. 


\title{
Moist versus Dry Barotropic Instability in a Shallow-Water Model of the Atmosphere with Moist Convection
}

\author{
Julien LAmbaerts, Guillaume Lapeyre, AND Vladimir Zeitlin \\ Laboratoire de Météorologie Dynamique/IPSL, Ecole Normale Supérieure/CNRS/UPMC, Paris, France
}

(Manuscript received 28 April 2010, in final form 6 January 2011)

\begin{abstract}
Dynamical influence of moist convection upon development of the barotropic instability is studied in the rotating shallow-water model. First, an exhaustive linear "dry" stability analysis of the Bickley jet is performed, and the most unstable mode identified in this way is used to initialize simulations to compare the development and the saturation of the instability in dry and moist configurations. High-resolution numerical simulations with a well-balanced finite-volume scheme reveal substantial qualitative and quantitative differences in the evolution of dry and moist-convective instabilities. The moist effects affect both balanced and unbalanced components of the flow. The most important differences between dry and moist evolution are 1) the enhanced efficiency of the moist-convective instability, which manifests itself by the increase of the growth rate at the onset of precipitation, and by a stronger deviation of the end state from the initial one, measured with a number of different norms; 2) a pronounced cyclone-anticyclone asymmetry during the nonlinear evolution of the moist-convective instability, which leads to an additional, with respect to the dry case, geostrophic adjustment, and the modification of the end state; and 3) an enhanced ageostrophic activity in the precipitation zones but also in the nonprecipitating areas because of the secondary geostrophic adjustment.
\end{abstract}

\section{Introduction}

Water vapor can influence the large-scale atmospheric dynamics through the latent heat release produced by condensation and precipitation processes. This particularly concerns the deep convection areas in the tropics. Yet, such influence is still not well understood. A partial explanation of this fact lies in complexity and intrinsic nonlinearity of the moist air thermodynamics (Emanuel 1994). For example, one of the most common cumulus parameterizations used in the general circulation models, the Betts-Miller scheme (Betts and Miller 1986), corresponds to convective quasi-equilibrium (QE) approximation (Emanuel et al. 1994) and expresses the precipitation and the related latent heat release in terms of relaxation of specific humidity toward the equilibrium profile, once moisture exceeds its saturation value. Thus, an essentially nonlinear threshold effect is introduced in the dynamics in the presence of moisture (acting as an active tracer).

A conceptual framework for studying dynamical role of moist processes was proposed in the pioneering paper

Corresponding author address: Vladimir Zeitlin, LMD-ENS, 24 rue Lhomond, 75005 Paris, France.

E-mail: zeitlin@lmd.ens.fr by Gill (1982), where a typical threshold nonlinearity was introduced in a simple shallow-water model of the atmosphere in the absence of nonlinear advection. Similar models were then extensively analyzed by Frierson et al. (2004), Stechman and Majda (2006), and Pauluis et al. (2008), in particular in the context of the precipitation fronts. Bouchut et al. (2009) recently proposed a model of this type, fully nonlinear in the hydrodynamic sector (i.e., with nonlinear advective terms): the moist-convective rotating shallow-water (mcRSW) model. It is important to stress that an efficient high-resolution finite-volume numerical implementation exists for this model and was successfully tested against the theoretical predictions.

The intrinsic nonlinearity of the moist processes forbids the standard "linear" thinking and related methods, like modal decomposition. This is especially important in the context of stability analysis, because linear stability has no sense anymore, and one should think in terms of nonlinear (Lyapunov) stability only. Yet, nonlinear analysis of moist instability is rarely performed (see, however, Rivière et al. 2009). Most often, humidity is supposed to simply reduce the static stability of the flow (e.g., Bennetts and Hoskins 1979; Emanuel et al.1987). A bulk of literature exists on the so-called convective instability of the second kind (CISK), which appears while linking the latent heat release and 
the low-level convergence of moisture (e.g., Charney and Eliassen 1964; Lindzen 1974). Most of such studies do not introduce the threshold effect or use the moisture evolution equation, with heating being linearly linked to the vertical velocity (e.g., Mak 1982, 1983). An investigation of such instability with CISK parameterization with conditional heating, which corresponds to the immediate relaxation limit for the Betts-Miller scheme, or the so-called strict quasi-equilibrium (SQE) approximation [this terminology was proposed by Emanuel et al. (1994)], was undertaken by Crum and Dunkerton (1992). In that particular case, the linear stability analysis still worked, but the instability was a direct consequence of the piecewise hyperbolic/ elliptic nature of the system (imaginary characteristic velocities in the "wet" regions). We should emphasize that "classical" instabilities in the presence of conditional heating for situations stable with respect to the CISK instability were not addressed in this context.

The dynamical influence of moisture and related phenomena is of obvious importance and is recurrently discussed in the context of predictability (e.g., Coutinho et al. 1994; Ehrendorfer et al. 1999; Langland et al. 1996; Rivière et al. 2009) in the GCMs framework. However, a systematic approach to the problem is still lacking.

The goal of the present paper is to identify and to quantify the differences induced by precipitation and latent heat release (with respect to the classical "dry" scenario) in the development and the saturation of the barotropic instability. In other words, we seek to identify and to quantify the role of the characteristic moist nonlinearity. The barotropic instability is a fundamental large-scale instability of the atmospheric flows. We will investigate it in the framework of the rotating shallow-water (RSW) model and its moist-convective counterpart (mcRSW) proposed by Bouchut et al. (2009). The RSW is chosen for simplicity and because efficient high-resolution numerical methods are available in the framework of this model both for linear stability analysis and for fully nonlinear simulations of the nonlinear stage of instability. Because no limitation is imposed on the divergence of the velocity field of the perturbation, we thus place ourselves in the context of divergent barotropic instability (Stern 1961; Lipps 1963; Philander 1976; Dunkerton 1990).

The mcRSW is the simplest physically and mathematically consistent model of large-scale horizontal motions with moisture, precipitation, and latent heat release. It is obtained by adding a relaxation sink due to precipitation in the otherwise locally conserved moisture equation and a corresponding convective sink in the mass conservation equation. Obviously, the most relevant to the atmosphere problem is the moist baroclinic instability. It may be studied, following the approach of the present paper, in the framework of the two-layer mcRSW. The latter is obtained along the same lines as the one-layer mcRSW in Bouchut et al. (2009) but considering three isobaric surfaces and the fluid layers between them, with moisture concentrated in the low-level flow. The convective mass sink of the onelayer model becomes a mass exchange term between the layers and leads to an additional drag in the upper-layer flow. This work is in progress and will be presented in a separate paper. Let us only mention that the simplest onelayer mcRSW used below may be obtained, as it should, from the (total) mass and momentum conserving twolayer model in the limit of infinitely thick upper layer.

Our approach is based on the exhaustive linear stability analysis in the dry model, initialization of high-resolution nonlinear simulation with thus identified most unstable modes both in dry and moist models, parallel dry and moist simulations of the evolution of the instability, and their comparisons. Such approach of studying saturation of instabilities has proven to be successful within the same model (albeit without active tracer) in preceding studies (Scherer and Zeitlin 2008; Gula and Zeitlin 2010). As a background flow, we take the classical Bickley jet. Its instabilities were extensively studied in the literature and in particular in the RSW context (Poulin and Flierl 2003). We consider this flow on the $f$ plane and aim mainly at the quasigeostrophic regime relevant for large-scale atmospheric dynamics, where it is known that the barotropic instability results in a vortex street formation. The dynamical effects of moisture are analyzed with special attention to the cyclone-anticyclone asymmetry, which was previously observed in quasigeostrophic moist dynamics (Lapeyre and Held 2004), and to the importance of the ageostrophic motions.

The paper is organized as follows: the mcRSW model is presented in section 2. Linear stability analysis of the Bickley jet in the dry model is presented in section 3 . The numerical setup is discussed in section 4 . The comparative moist and dry simulations are then studied in the quasilinear (section 5) and nonlinear (section 6) regimes with special emphasis on the growth of the perturbation and the vortex street formation. Note that, although moisture is always present in our simulations, we will often abusively call the simulations where the precipitation term is switched off and moisture is just a locally conserved passive scalar as dry, and simulations with allowed precipitation as moist. The parameter dependence of our results is discussed in section 7. Conclusions and discussion follow in section 8 .

\section{The mcRSW model}

The mcRSW model proposed in Bouchut et al. (2009) may be derived by vertical averaging of primitive equations in pseudoheight coordinates (cf. Hoskins and Bretherton 

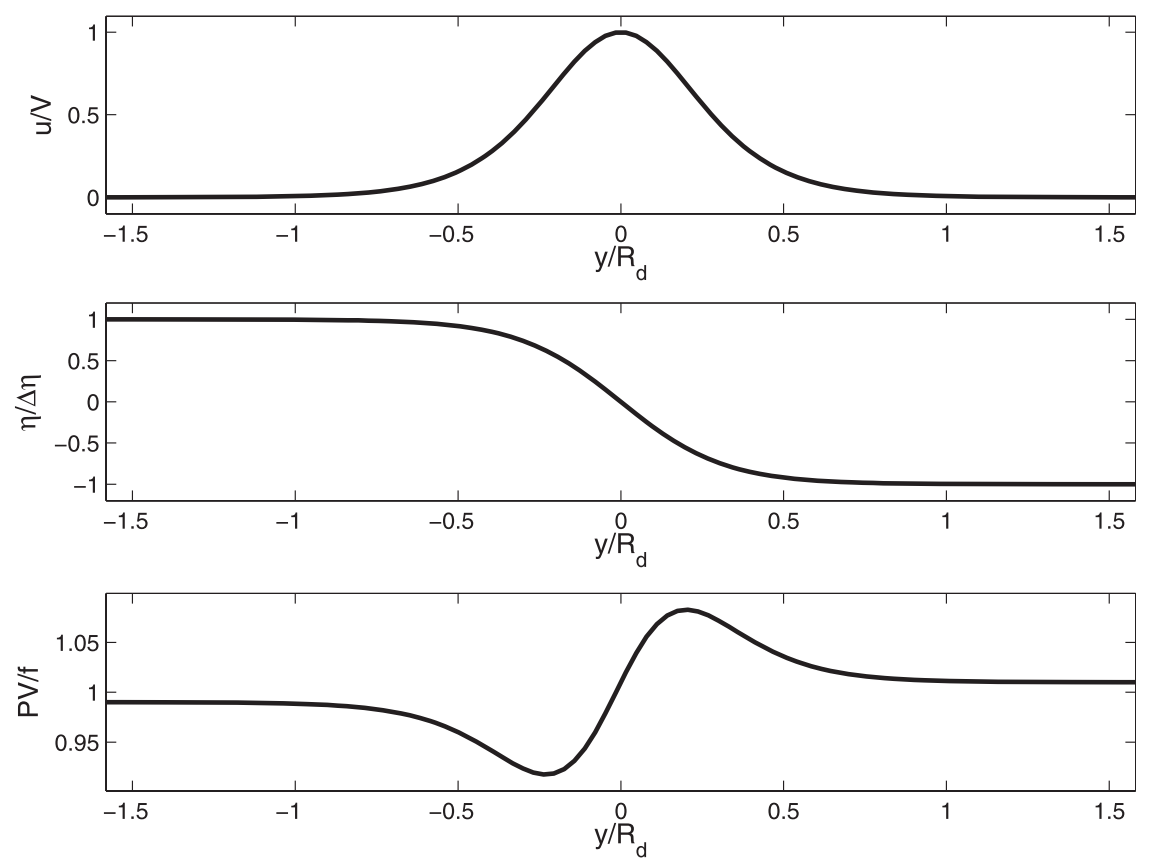

FIG. 1. Bickley jet: profiles of (top) $u$, (middle) $\eta$, and (bottom) PV (Ro $=0.1, \mathrm{Bu}=10, H_{0}=1$, $f=10$, and $L=0.1)$.

1972) between an upper isobaric surface $z_{2}$ (=constant) and a bottom material surface $z_{1}(x, y, t)$ with constant geopotential $\left(\left.\varphi\right|_{z_{1}}=\right.$ constant $)$. Precipitation enters as a moisture sink $P$ in the conservation law for specific humidity, and latent heat release is linked to additional vertical (convective) velocity $W$ at $z_{2}$. Thus, $W$ represents the moist convection, because one can show that $W$ is directly related to $P: W=\beta P$, where $\beta$ is a positive coefficient, as follows from the conservation of the moist enthalpy for the isentropic layer $\left(z_{1}, z_{2}\right)$. The system is not supposed to model the whole troposphere but rather its lower part. A more realistic model contains two or more layers with convective exchanges between them and will be presented elsewhere (Lambaerts et al. 2011). One can show that the one-layer model below results, as it should, from the two-layer one in the limit of infinitely thick second layer.

The equations of the model are

$$
\begin{aligned}
\partial_{t} \mathbf{v}+(\mathbf{v} \cdot \nabla) \mathbf{v} & =-g \nabla h-f \hat{z} \times \mathbf{v}, \\
\partial_{t} h+\nabla \cdot(\mathbf{v} h) & =-\beta P, \quad \text { and } \\
\partial_{t} Q+\nabla \cdot(\mathbf{v} Q) & =-P,
\end{aligned}
$$

with

$$
P=\frac{Q-Q_{s}}{\tau} H\left(Q-Q_{s}\right)
$$

where $\mathbf{v}=(u, v)$ is the horizontal velocity field, $f$ is the Coriolis parameter, $g$ is the acceleration due to gravity, $h=$ $z_{2}-z_{1}$ is the layer thickness, $Q=\int_{z_{1}}^{z_{2}} q d z$ is the specific humidity integrated over the layer, and $H(\cdot)$ is the Heaviside function. Precipitation is expressed according to the QE closure, with conditional relaxation of $Q$ at a characteristic time $\tau$ to its saturation value $Q_{s}$, taken to be constant for simplicity.

The moist enthalpy in the model is expressed as $m=h-$ $\beta Q$, and it is indeed conserved and is easy to check. The moist enthalpy balance prevails over the standard energy balance, where precipitation induces an effective dissipation. The latter is related to the mass nonconservation in the layer due to the convection sink. We should emphasize that the moist enthalpy conservation limits the mass loss, provided initial moist enthalpy is everywhere positive. These properties are discussed at length in Bouchut et al. (2009).

\section{Bickley jet: Linear stability analysis in the dry case}

Following Poulin and Flierl (2003), we analyze in this section the barotropic instability of a geostrophically balanced Bickley jet (Fig. 1) on an $f$ plane with $f=$ constant,

$$
\left\{\begin{array}{l}
\bar{u}=\frac{g \Delta \eta}{f L} \operatorname{sech}^{2}\left(\frac{y}{L}\right), \\
\bar{v}=0 \\
\bar{h}=H_{0}+\bar{\eta}=H_{0}-\Delta \eta \tanh \left(\frac{y}{L}\right),
\end{array}\right.
$$



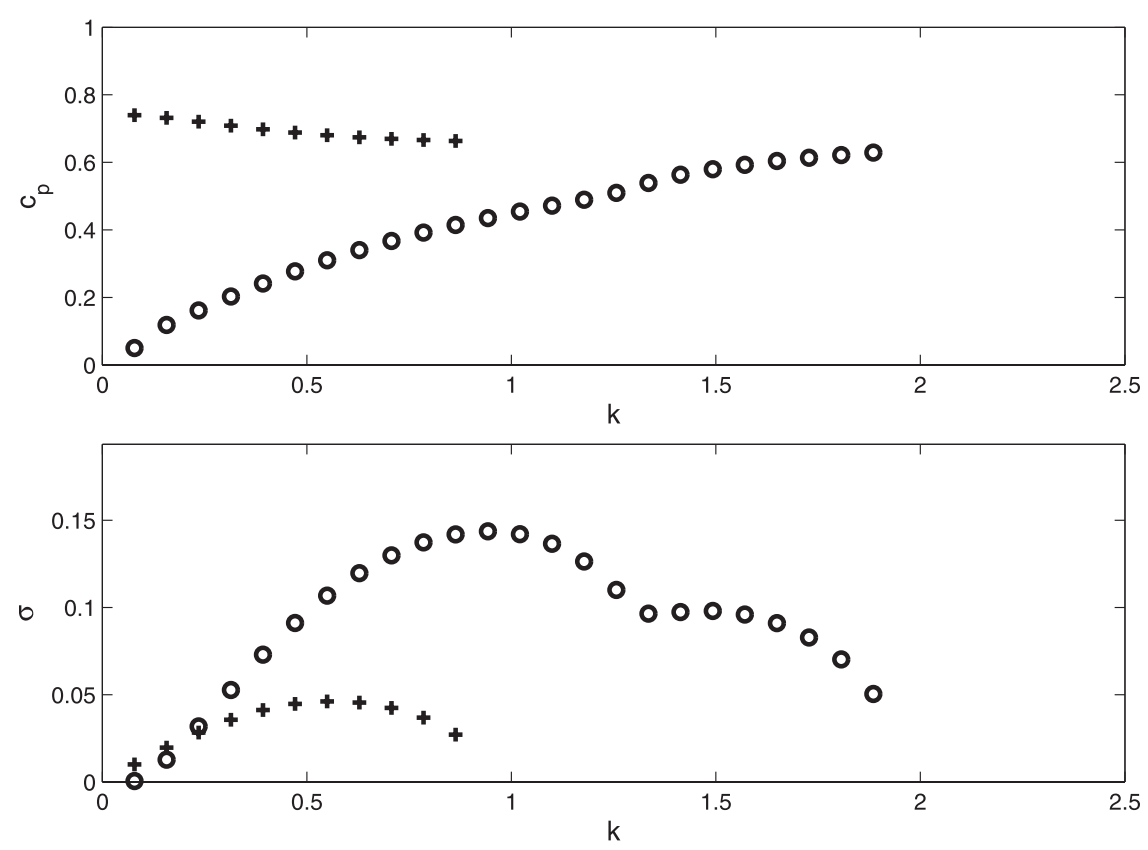

FIG. 2. Dimensionless (top) $c_{p}$ and (bottom) $\sigma$ of the unstable modes of the Bickley jet of Fig. 1, as functions of $k$. Circles and crosses correspond to two different unstable modes; $N=200$ $L_{y}=2$, and $L=0.1$.

where $L$ is the length scale of the jet and $\Delta \eta$ is a constant. One can easily verify that (3.1) is indeed a stationary solution of (2.1)-(2.3) for uniform unsaturated moisture field $\left(Q=Q_{0}=\right.$ constant $\left.<Q_{3}\right)$. Note that shallow-water equations on the $f$ plane are symmetric with respect to rotations so zonal and meridional coordinates are interchangeable, with atmospheric jets being mostly zonal.

The jet is characterized by two dimensionless numbers: the Rossby number Ro $=V / f L$ and the Burger number $\mathrm{Bu}=R_{d}^{2} / L^{2}=g H_{0} / f^{2} L^{2}$, where $R_{d}=\sqrt{g H_{0}} / f$ is the deformation radius and $V=g \Delta \eta / f L$ is the maximum velocity of the jet. We start with the linear stability analysis of a dry jet. This will allow us to obtain the exact structure of unstable modes, which will be superimposed onto the jet to initialize the nonlinear simulations. The structure of unstable modes also gives important clues for understanding the mechanisms of the instability and the evolution of the moisture field.

The dry $(P=0)$ equations (2.1) and (2.2) are linearized around the stationary jet [(3.1)]. Dimensionless equations for the perturbation $(u, v, \eta)$ are

$$
\begin{gathered}
\operatorname{Ro}\left(\partial_{t} u+\bar{u} \partial_{x} u+v \partial_{y} \bar{u}\right)-v+\partial_{x} \eta=0, \\
\operatorname{Ro}\left(\partial_{t} v+\bar{u} \partial_{x} v\right)+u+\partial_{y} \eta=0, \quad \text { and }
\end{gathered}
$$

$\operatorname{Ro}\left[\partial_{t} \eta+\bar{u} \partial_{x} \eta+\bar{\eta} \partial_{x} u+\partial_{y}(v \bar{\eta})\right]+\operatorname{Bu}\left(\partial_{x} u+\partial_{y} v\right)=0$.
Using the Fourier decomposition, $(u, v, \eta)=(\hat{u}, i k \hat{v}, \hat{\eta})$ $\exp [i(k x-\omega t)]$, we obtain an eigenvalue problem: $\mathcal{L} \mathbf{a}=$ ca, with $\mathbf{a}=(\hat{u}, \hat{v}, \hat{\eta})$ and

$$
\mathcal{L}=\left[\begin{array}{ccc}
\bar{u} & \partial_{y} \bar{u}-\frac{1}{\mathrm{Ro}} & \frac{1}{\mathrm{Ro}} \\
\frac{-1}{\mathrm{Ro}^{2}} & \bar{u} & \frac{-1}{\mathrm{Ro}^{2}} \partial_{y} \\
\bar{\eta}+\frac{\mathrm{Bu}}{\mathrm{Ro}} & \partial_{y} \bar{\eta}+\left(\bar{\eta}+\frac{\mathrm{Bu}}{\mathrm{Ro}}\right) & \bar{u}
\end{array}\right] .
$$

The eigenvalues $c=\omega / k$ and corresponding eigenvectors of this problem are found with the help of the collocation method (Trefethen 2000). Below, we will not explore the $(\mathrm{Ro}, \mathrm{Bu})$ space, which was already done by Poulin and Flierl (2003), but we will focus only on the quasigeostrophic regime relevant for large-scale atmospheric motions, taking the following typical values of parameters: $\mathrm{Ro}=0.1$ and $\mathrm{Bu}=10$. The corresponding dispersion diagram is presented in Fig. 2 for a calculation with the number of Chebychev collocation points $N=200$, the domain size $L_{y}=2$, and $L=0.1$. The dimensionless phase velocity $c_{p}=\operatorname{Re}(c)$ and the growth rate $\sigma=\operatorname{Im}(c) k$ are displayed in the figure as functions of the dimensionless wavenumber $k$ for the unstable modes (stable modes are not shown). The unstable modes appear in the band $c_{p} \in$ $[0,1]$. They can be interpreted as Rossby waves propagating on the background of strong potential vorticity (PV) gradients of the underlying jet. It should be stressed 
that one of the difficulties of the stability analysis by the collocation method is the appearance of pseudomodes with critical levels $y=y_{c}$, defined by $\bar{u}\left(y_{c}\right)=c_{p}$, inside the jet. They are singular and are removed by the gradientlimiter method (see Scherer and Zeitlin 2008). Two types of unstable modes are clearly visible in Fig. 2. The less unstable branch with wavenumbers between $k \approx 0$ and $k \approx 1$ corresponds to faster waves (cf. Fig. 2, top). For larger $k$, it splits in two stable $(\sigma=0)$ branches corresponding to dipolar Rossby modes. The more unstable branch, with wavenumbers between $k \approx 0$ and $k \approx 2$, corresponds to slower waves. The maximum growth rate is $\sigma_{\max }=0.142$ at $k=0.942$. This branch for larger $k$ splits in two stable branches associated with monopolar Rossby modes. ${ }^{1}$ The less unstable mode has varicose and the more unstable mode sinuous structure in the cross-jet direction, consistent with the earlier studies by Hazel (1972). It is worth noting that the two unstable modes have critical levels on opposite sides of the inflection point of the background jet and hence will be affected differently on the beta plane (which is out of the scope of the present paper).

If Fig. 2 (bottom) is compared with Fig. 3 (panel 1) of Poulin and Flierl (2003), which corresponds to the same values of $\mathrm{Ro}$ and $\mathrm{Bu}$, qualitative and quantitative agreement between the curve of Poulin and Flierl and ours is perfect. The spatial structure of the most unstable mode in our calculation corresponds to the third mode $n=3$ for an $x$ domain of size $L_{x}=2: k=\left(2 \pi n / L_{x}\right) L$, identified by Poulin and Flierl. However, they apparently did not resolve the less unstable mode, apart from its long-wave tail, which was confused with the tail of the upper curve.

Figure 3 represents the spatial structure of the most unstable mode. It is close to the geostrophic balance and is composed of positive/negative interface deviations alternating along the background jet and centered over the maximum of the PV gradient of the jet (cf. Fig. 1, bottom). This is typical for Rossby waves. The main interface deviations are sided by weaker "wings" slightly shifted along the jet. The geostrophic balance is almost attained, but a weak divergence subsists. ${ }^{2}$

In what follows, we will use the most unstable mode identified by this analysis to initialize the nonlinear simulations of the evolution of the barotropic instability of the Bickley jet both in the dry and in the moist/ precipitating cases.

\footnotetext{
${ }^{1}$ It should be noted that the amplitude of the dip of the $\sigma$ curve at $k \approx 1.7$ is sensitive to the resolution: at higher $N$ it gradually smoothens.

${ }^{2}$ Weak values of divergence are sensitive to the method of numerical differentiation; centered differences are used throughout the paper.
}

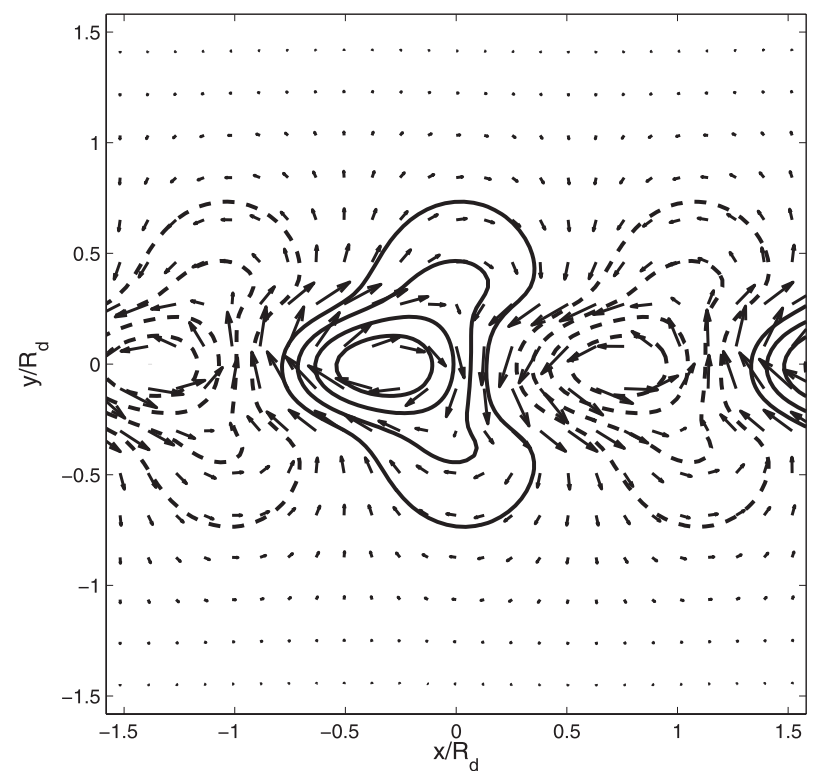

FIG. 3. Spatial structure of the most unstable linear mode of the Bickley jet: thickness (contours) and velocity (arrows) fields. The full lines correspond to positive values, and the dashed lines to negative values.

\section{Numerical setup}

For the nonlinear simulations of nonlinear development of the instability we use the high-resolution, well balanced, entropy-satisfying finite-volume numerical scheme for RSW equations (Bouchut et al. 2004; Bouchut 2007). This scheme has the following properties, which are important in the present context: 1 ) it is well balanced (in a sense of balancing pressure gradients with a stiff source, the Coriolis force), which allows to maintain the geostrophic equilibrium and to preserve PV with good accuracy (in the absence of dissipation); 2) it is virtually dissipationless, with (numerical) dissipation acting exclusively in the strong gradient zones (shocks) and by construction diminishing energy (entropy inequalities are built in the scheme, with entropy being energy in the RSW context; cf. Whitham (1974). In the moist configuration, an equation for a locally conserved tracer, specific humidity, is added into the scheme, and conditional relaxation law for precipitation is introduced at the end of each iteration. The scheme in the dry configuration was extensively tested (Bouchut et al. 2004; Bouchut et al. 2005; Scherer and Zeitlin 2008; Gula and Zeitlin 2010) and was recently benchmarked against the $1 \mathrm{D}$ analytical results in the moist-convective version (Bouchut et al. 2009).

To remain close to the work of Poulin and Flierl (2003), which we will use for comparison with our results, as was done above for linear stability analysis, we choose for the nonlinear simulation a spatial domain $L_{x}=L_{y}=2$, with 
periodic and Neumann boundary conditions in the $x$ and $y$ directions, respectively. The resolution is fixed at $N_{x}=$ $N_{y}=200$ with equally spaced points in each direction. The jet parameters are taken to be the same as in Poulin and Flierl (2003), $H_{0}=1, f=10$, and $L=0.1$, for the sake of comparison.

The barotropic instability is generated by superimposing the most unstable mode $(v, \eta)$ of small amplitude upon the balanced jet $(\bar{v}, \bar{h})$. The initial moisture field is chosen to be uniform and unsaturated $Q\left(t_{0}\right)=Q_{0}<Q_{s}$. Note that, if saturated configuration $Q_{0}=Q_{s}$ is taken as initial condition, it immediately triggers precipitation in the convergence areas so that comparison with the dry linear analysis of section 3 is not possible even for initial times.

To be able to observe the effects of precipitation and latent heat release during the evolution of the instability, one must choose the initial saturation gap $Q_{s}-Q_{0}$ to be smaller than the maximum gap $Q(t)-Q_{0}$ observed during the evolution of the dry instability $(P=0)$,

$$
Q_{0}<Q_{s}<Q_{P=0}^{\max }
$$

when moisture is just locally conserved. The moisture relaxation is chosen to be rapid, mimicking the immediate relaxation limit $(\tau \rightarrow 0), \tau=\gamma \Delta t$, where $\gamma=5$ and $\Delta t$ is the time step of the numerical scheme determined as the minimum between a specific value allowing to respect the Courant-Friedrich-Levy condition $\Delta t^{\mathrm{CFL}}$ and a maximum value $\Delta t^{\max }=2 \times 10^{-3}$ determining the resolution.

As was shown in Bouchut et al. (2009), in the limit $\tau \rightarrow$ 0 , our parameterization of the moist convection coincides with the CISK with conditional heating or strict quasiequilibrium one, with corresponding changes in the gravity wave speed in the precipitating regions. The observations show that the values of the moist gravity wave speed in the atmosphere are $c_{m} \approx 0.3 c_{d}$ (Wheeler and Kiladis 1999), where $c_{d}=\sqrt{g H_{0}}$ is the dry linear gravity wave speed. For $\beta=1$, we therefore choose $Q_{s}=1-\left(c_{m} / c_{d}\right)^{2}=0.9$, which corresponds to the value used by Frierson et al. (2004), Stechman and Majda (2006), and Pauluis et al. (2008) in most of their numerical experiments with the linear version of the model. Thus, $Q_{0}$ must be chosen correspondingly, taking into account (4.1); see section 7 .

\section{Quasi-linear regime}

In this section, the evolution of the instability is analyzed in the quasi-linear regime in which the perturbation grows quasi exponentially: $t \in\left[0,100 T_{i}\right]$, where $T_{i}=f^{-1}$, which will be abusively called below inertial period, with the true inertial period being $2 \pi f^{-1}$. The parameters are chosen to trigger precipitation in this regime (at $t \approx 28 T_{i}$ ) in the moist simulation: $Q_{0}=0.899$ and $Q_{s}=0.9$, which is compared below to the dry one.

\section{a. Initial stage: The geostrophic adjustment}

For $t \approx T_{i}$, the ageostrophic (unbalanced) part of the perturbation is evacuated because of emission of inertiagravity waves (IGW) as can be seen in the divergence field $\delta=\nabla \cdot \mathbf{v}$, but also in $\eta$, in Fig. 4. This is an example of the geostrophic adjustment at small Ro (cf., e.g., Zeitlin 2008). It results from discretization errors and forcing by nonlinear terms (the unstable mode is retrieved with finite accuracy in Chebyshev collocation points and is not a solution of full nonlinear equations). The adjustment is better visible in the $y$ direction because the perturbation possesses a unique $x$ harmonic $k$ such that short IGW are quasi unidirectional: $k \ll l$, where $k$ and $l$ are $x$ and $y$ wavenumbers, respectively. The shortest waves propagate with the dry linear gravity wave speed $c_{d}=\sqrt{g H_{0}}$. Note that the adjustment process is maintained by the exponential growth of the perturbation. Consequently, the IGW emission continues.

The adjustment process affects the initially uniform moisture field; compare Fig. 4, bottom, and Fig. 5, where the anomaly $q=Q-Q_{0}$ with respect to initially uniform background moisture $Q_{0}$ is presented. Ageostrophic perturbations at the periphery (wings) of the unstable mode create moisture anomalies. At the same time, these anomalies are advected by the balanced part of the unstable mode, which can be considered quasi stationary during this initial period, and form coherent spatial structures. Naturally, the results of dry and moist simulations coincide as long as precipitation is not triggered.

Note that, to see the influence of precipitation on the geostrophic adjustment process, one should start from a supersaturated state, as in a recent study of Dias and Pauluis (2010) performed in the framework of the linear model of Neelin and Zeng (2000).

\section{b. Growth rates}

As was already mentioned in the introduction, the standard linear stability analysis of the moist-convective system (2.1)-(2.3) is impossible. We therefore pass to direct diagnostics of the Lyapunov stability on the basis of the nonlinear simulation. The growth of the same initial perturbation in dry and moist simulations can be compared in this setting along with the linear growth rate.

The growth rate $\sigma$ of the perturbation is estimated in a given norm. The dry energy norm

$$
s^{2}(t)=\iint d x d y\left(H_{0} \frac{\mathbf{v}^{2}}{2}+g \frac{\eta^{2}}{2}\right)
$$



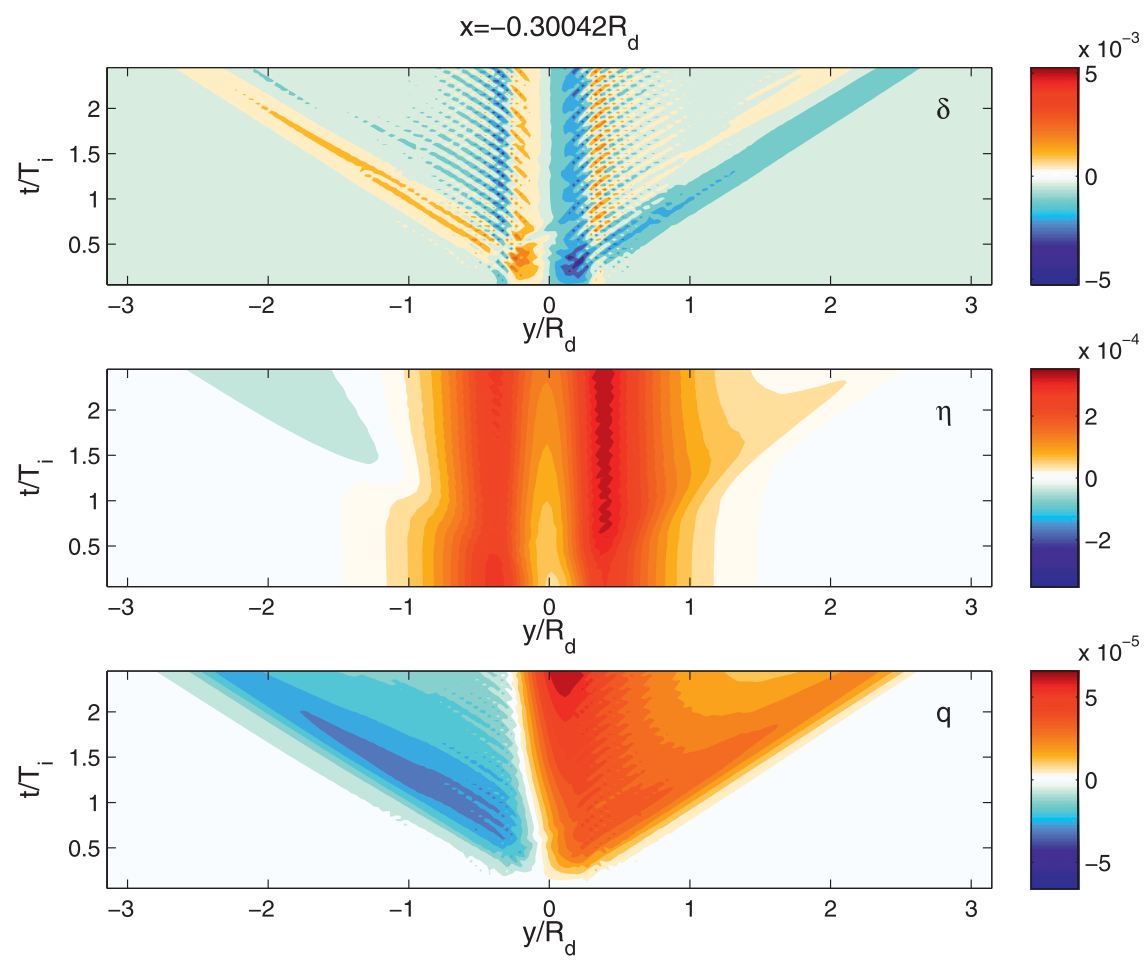

FIG. 4. Hovmöller diagrams of (top) $\delta=\nabla \cdot \mathbf{v}$, (middle) $\eta$, and (bottom) $q=Q-Q_{0}$ at the initial stage of evolution of the instability.

seems to be appropriate to quantify the instability. For the system (2.1)-(2.3) with linearization of advective and flux terms over the state of rest $(\overline{\mathbf{v}}=0=\bar{\eta})$, we get

$\frac{d}{d t} s^{2}=-g H_{0} \iint d x d y \nabla \cdot(\eta \mathbf{v})-g \beta \iint d x d y \eta P$.

This formula shows that the dry energy norm $s^{2}$, which is otherwise conserved (for the isolated system), will be affected by precipitation and is sensitive to the sign of the perturbation $\eta$ in the precipitating regions, as was already discussed in Gill (1982) and Lapeyre and Held (2004).

Another possible choice, as also discussed in Gill (1982) and Lapeyre and Held (2004), is the moist energy norm $s_{m}^{2}$, which has an advantage to be conserved (over a saturated state of rest) even in the precipitating regions in the immediate relaxation limit $\tau \rightarrow 0$,

$$
\begin{aligned}
s_{m}^{2}(t)= & \iint d x d y\left[H_{0} \frac{\mathbf{v}^{2}}{2}+\frac{g H_{0}}{H_{0}-\beta Q_{s}} \frac{(\eta-\beta \tilde{q})^{2}}{2}\right. \\
& \left.+\frac{g \beta H_{0}}{Q_{s}} \frac{(\tilde{q})^{2}}{2}\right]
\end{aligned}
$$

where $\tilde{q}=Q-Q_{s}$ denotes the moisture deficit. The dry potential energy entering $s^{2}$ is replaced here by the pseudoenergy depending on the moist enthalpy $\eta-\beta \tilde{q}$ and moisture deficit $\tilde{q}$. However, $s_{m}^{2}$ is not well suited for the initial growth stage because, before growing, the moisture anomalies must emerge first from the unsaturated initial state with uniform $\tilde{q}=Q_{0}-Q_{s}$. This is why we will use the dry energy norm in this section.

The exponential growth rate associated to the normalized dry energy norm,

$$
\sigma(t)=\frac{1}{2} \frac{d}{d t} \ln \left[\frac{s^{2}(t)}{s^{2}\left(t_{0}\right)}\right]
$$

is presented in Fig. 6 for dry (solid line) and moist (dashed line) simulations. In the dry simulation, the growth rate starts with an approximately constant value, $\sigma_{0} \approx 0.12$, and then slowly decreases. Its numerical value at initial stage is slightly weaker than the one expected from the dry linear analysis $(\sigma=0.142$; see section 3$)$. This can be explained by the fact that the numerical scheme is nonlinear and generates an energy flux from the main unstable mode toward the higher ones.

In the moist simulation, the growth rate suddenly increases when precipitation is triggered $\left(t \approx 28 T_{i}\right)$. This means that precipitation and moist convection temporary, as well as globally, enhance the instability even if this effect is weak (only a $10 \%$ increase of the growth rate). According to the source term in (5.2), this is due to the dominating negative thickness anomaly in the precipitating region [taking the Bickley jet instead of the state of 

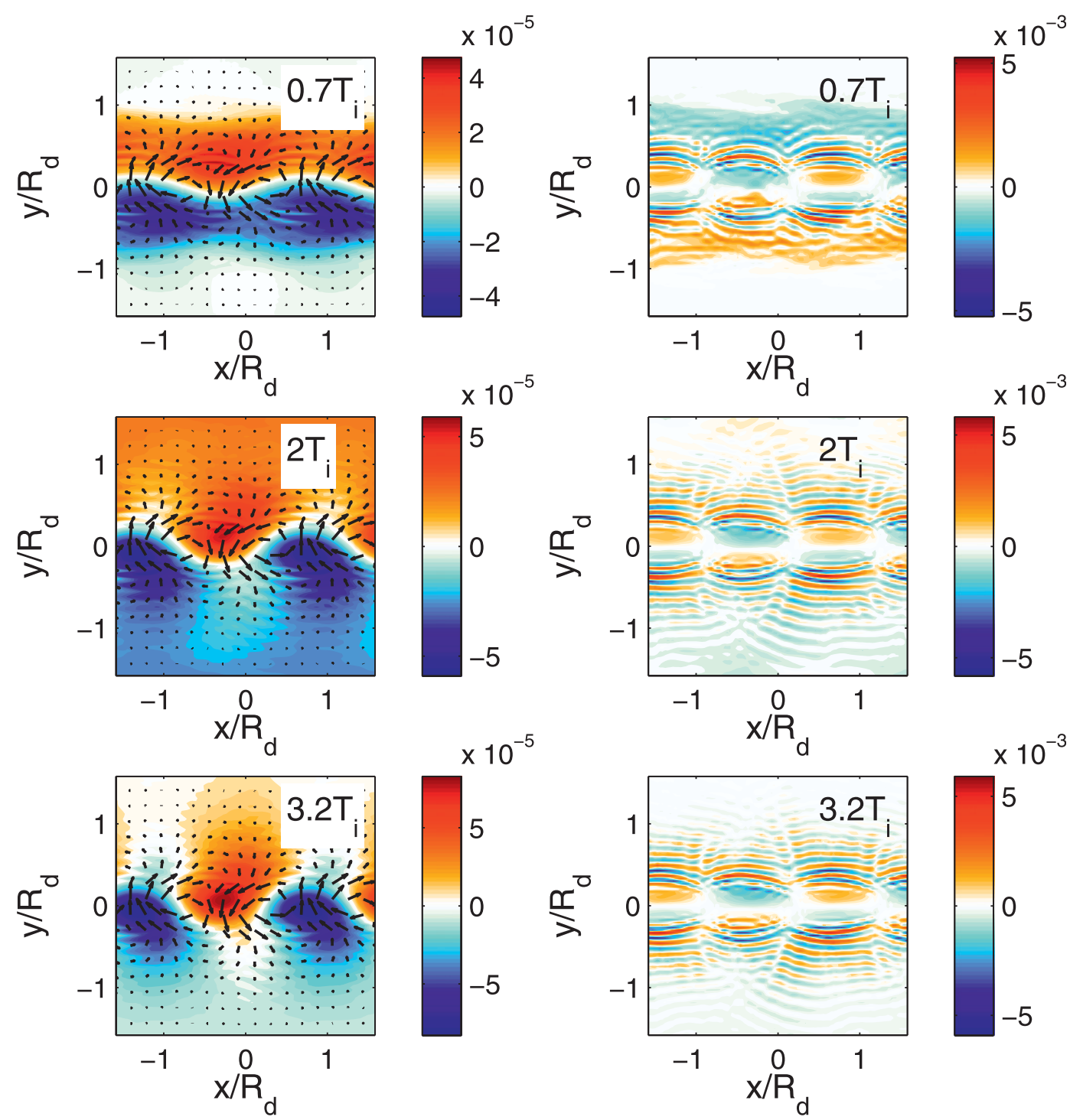

FIG. 5. Evolution of $q$ (colors) and (left) of $\mathbf{v}$ (arrows) and (right) of $\delta$ at initial times of the development of the instability.

rest as a background: $\eta \rightarrow \bar{\eta}+\eta$ does not essentially change (5.2)]. Note that precipitation affects at the first place the potential energy contribution into the norm (see below).

\section{Fully nonlinear regime}

Because of initial quasi-exponential growth (cf. Fig. 6), the nonlinear terms become significant and lead to reorganization of the growing perturbation. A vortex street is progressively formed, tending to reach a new geostrophically balanced state. The mechanism of its formation is analyzed and compared in the dry and moist cases in this section. It is to be stressed that the results of our dry simulation are in perfect agreement with corresponding results of Poulin and Flierl (2003).

\section{a. Quasigeostrophic (balanced) slow dynamics and vortex street formation}

\section{1) DRY SIMULATION}

For longer times $t>100 T_{i}$, the perturbation that continues to grow is distorted by the interaction with the underlying jet. Figure 7a represents the nonlinear evolution of the perturbation in the dry simulation. It should be noted that the color scale changes in time because of the development of the instability. The velocity vectors follow the isolines of $\eta$, the geostrophic streamfunction, with relative vorticity $\partial_{x} v-\partial_{y} u \approx g / f \nabla^{2} \eta$. At the early 


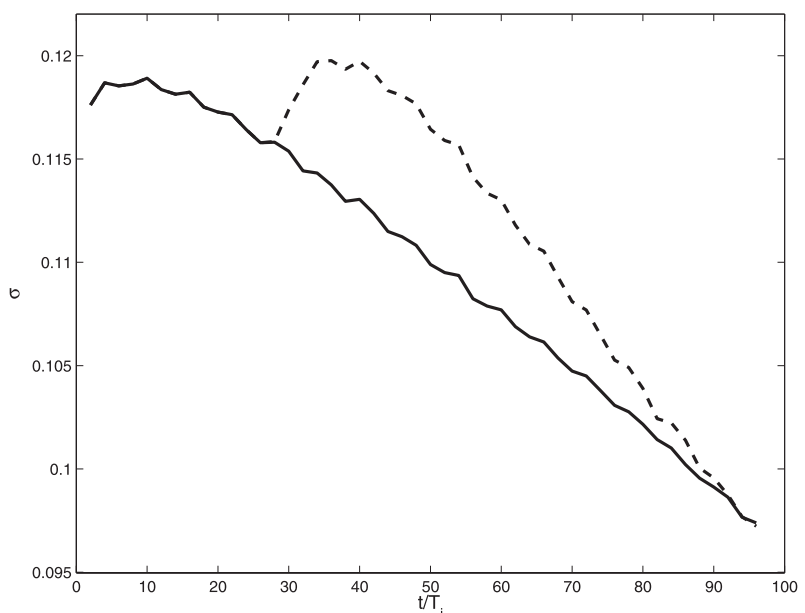

FIG. 6. Evolution of $\sigma$ associated with $s^{2}(t)$ for the dry (solid) and the moist (dashed) simulations.

stages $\left(t=40 T_{i}\right)$ positive (negative) perturbation vorticity centers (PVC), which are easy to identify in Fig. 7a by characteristic velocity patterns, are located in the middle of the jet. They gradually change their initial positions and are laterally displaced $\left(t=140 T_{i}\right.$ and $t=$ $240 T_{i}$ ). Thus, they start to slow down the jet at its center and accelerate it at the borders. The original peripheral wings of the unstable mode (cf. Fig. 3) thus disappear. They are replaced by alternating strong and weak PVCs at each side of the jet.

The areas in between the strong PVCs can be interpreted as slowdown and those inside the weak PVCs as vortex. As a consequence, a vortex street forms along the jet. Its emergence is clearly seen in Fig. 8a, where the evolution of the total (jet plus perturbation) relative vorticity $\zeta=\bar{\zeta}+\partial_{x} v-\partial_{y} u$ in the dry simulation is displayed. One can observe the intensification of vorticity in the vortex areas, the tilting of the strong vorticity gradient between the slowdown areas, the reconnection of the original vorticity isolines, and finally a stabilization of the vortex street (cf. Fig. 7a).

\section{2) Moist-CONVECTIVE Simulation}

It is expected that precipitation modifies the dry scenario of the vortex street formation presented above, because $\mathrm{PV}$ is not a Lagrangian invariant in the presence of precipitation $P>0,{ }^{3}$

$$
\left(\partial_{t}+\mathbf{v} \cdot \nabla\right) \frac{\zeta+f}{h}=\frac{\zeta+f}{h^{2}} \beta P .
$$

\footnotetext{
${ }^{3}$ It is worth noting that (6.1) does not contradict the impermeability PV theorem by Haynes and McIntyre (1987), because the layer boundaries are isobars, and not isentropes. Moreover, the upper one allows for the mass exchanges.
}

Thus, precipitation should affect the evolution of the perturbation tending to reach a new equilibrium state. Note that Lagrangian conservation of the moist equivalent PV follows from the conservation of the moist enthalpy $m$,

$$
\left(\partial_{t}+\mathbf{v} \cdot \nabla\right) \frac{\zeta+f}{h}=0
$$

Figure $7 \mathrm{~b}$ displays the evolution of $\eta$ in the moist simulation. Compared to the dry case of Fig. 7a, the nonlinear distortion of the perturbation now arises with a progressive nonuniform decrease of the layer thickness $\eta$. Of course, such decrease is expected by (2.2). However, the nonuniformity does not exactly correspond to the precipitating regions delimited by the thick contours in Fig. 7b. It should be noted that the vortex pattern of Fig. $7 \mathrm{~b}$ becomes better exposed if the secular trend in the zonal mean thickness due to precipitation is subtracted (not shown). However, subtracting the zonal mean, which is time dependent in the moist and time independent in the dry simulations, does not make much sense for comparison. In fact, one observes that cyclonic (anticyclonic) motions with $\nabla^{2} \eta>0\left(\nabla^{2} \eta<\right.$ 0 ) are progressively intensifying (weakening) close to the precipitating regions, particularly at $t \approx 240 T_{i}$ and $t \approx$ $340 T_{i}$. Such behavior can be easily explained in the quasigeostrophic approximation. For $\mathrm{Ro} \rightarrow 0$ and $\mathrm{Bu} \sim O(1)$, the system (2.1)-(2.2) in terms of dimensionless variables becomes

$$
\begin{aligned}
\left(\partial_{t}+\mathbf{v}^{(0)} \cdot \nabla\right)\left(\nabla^{2} \psi-\psi\right) & =\beta P \quad \text { and } \\
\left(\partial_{t}+\mathbf{v}^{(0)} \cdot \nabla\right)\left(\tilde{q}-Q_{s} \nabla^{2} \psi\right) & =-P,
\end{aligned}
$$

where $\mathbf{v}^{(0)}=\left(-\partial_{y} \psi, \delta_{x} \psi\right)$ is the geostrophic velocity, $\psi=\bar{\eta}+\eta$, and $\tilde{q}$ is the moisture deficit. These equations correspond to the conservation of PV $\left(\nabla^{2} \psi-\psi\right)$ and of moisture, respectively. They are obtained by considering typical nonlinearities in the $h$ and $Q$ equations to be on the order of the Rossby number and supposing quasi saturation, $\psi \sim \tilde{q} \ll 1$.

Therefore, the PV of the fluid columns that pass through the precipitating regions increases. Furthermore, in the limit of immediate relaxation $(\tau \rightarrow 0)$ of moisture in the precipitating regions, we can consider $\tilde{q} \approx 0$, such that (6.4) becomes

$$
Q_{s}\left(\partial_{t}+\mathbf{v}^{(0)} \cdot \nabla\right)\left(\nabla^{2} \psi\right) \approx P_{\tau \rightarrow 0}>0,
$$

which clearly shows the increase of the geostrophic vorticity in the precipitation regions.

Consequently, asymmetry between strong cyclonic and anticyclonic PVCs of the initial perturbation (Fig. 9a) 


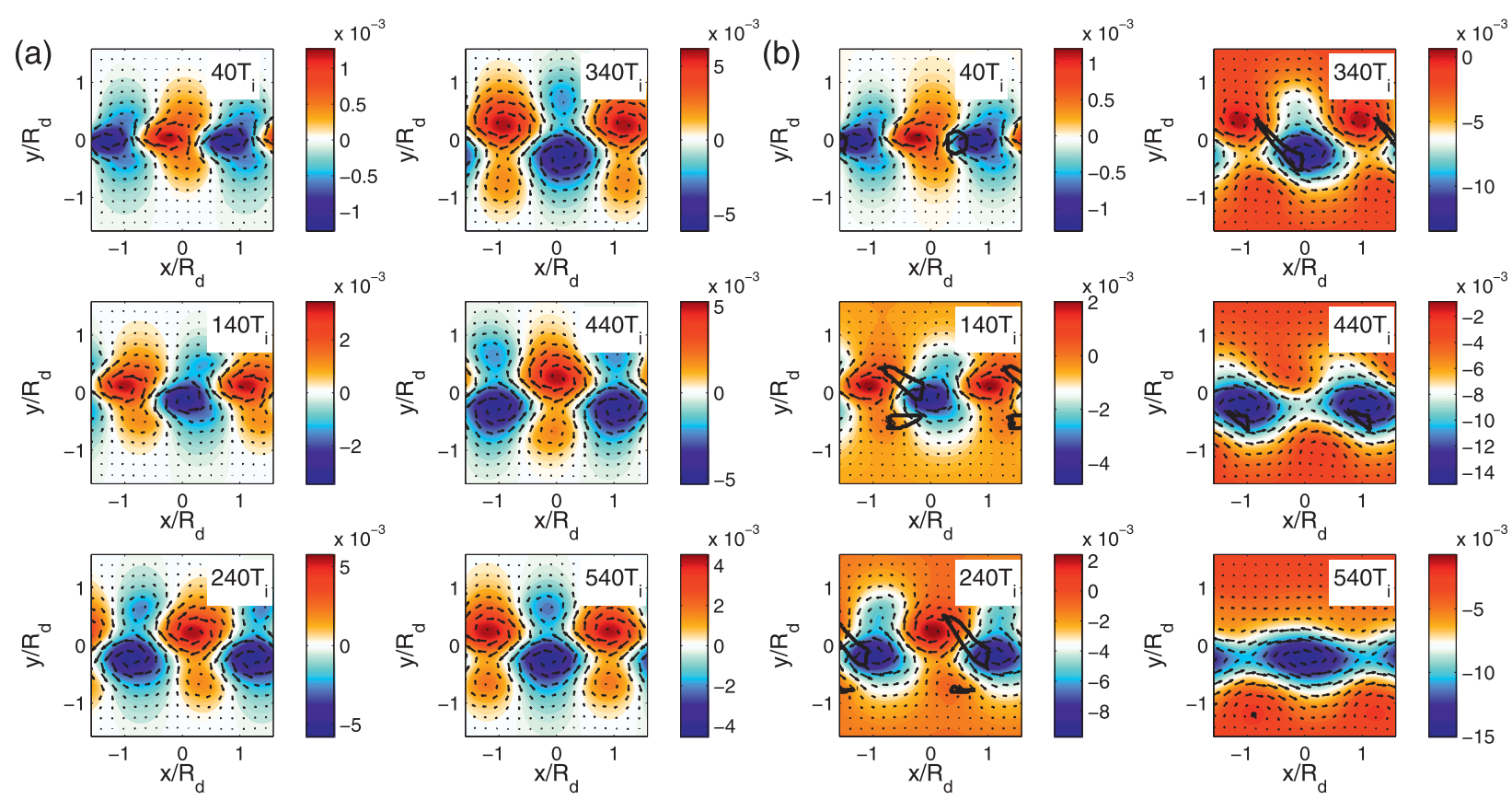

FIG. 7. Nonlinear evolution of $\eta$ (colors) and $\mathbf{v}$ (arrows) of the perturbation in the (a) dry and (b) moist-convective simulations. In (b), thick contours delimit the precipitation zones. Note that we change the color scale from panel to panel to better distinguish the structure of the flow.

develops because of precipitation. Advection of the weaker anticyclonic PVCs by stronger cyclonic PVCs induces a tilting effect upon the original dry configuration (Fig. 9b). Finally, the configuration with a dominant cyclonic PVC adjusts to a new equilibrium state (Fig. 9c).
Figure $8 \mathrm{~b}$ gives the evolution of $\zeta$ in the moist-convective simulation. The role of PVC in the formation of the vortex street becomes clear from the figure: a strong cyclonic PVC of Fig. $7 \mathrm{~b}\left(t=540 T_{i}\right)$ both slows down the jet and maintains the cyclonic vortex. The asymmetry between the (a)
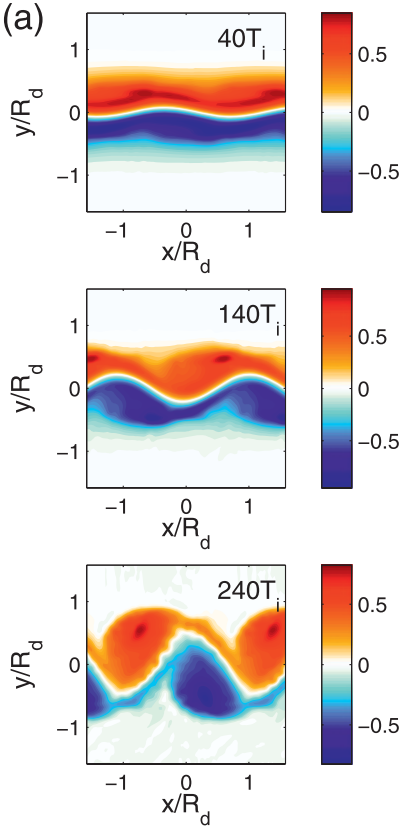
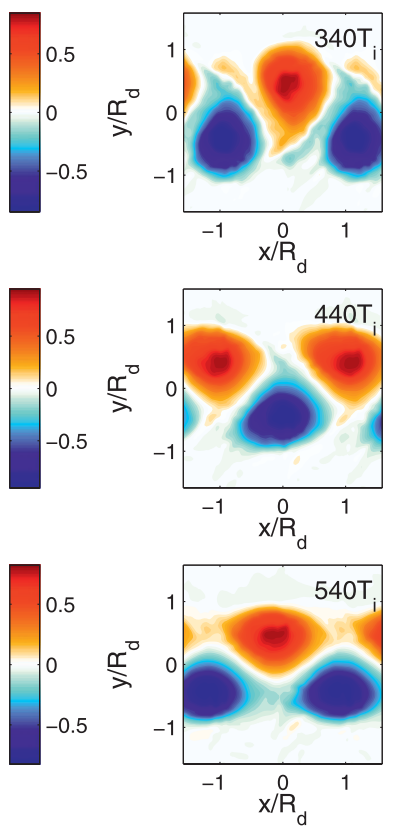
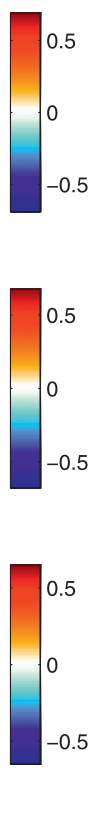

(b)
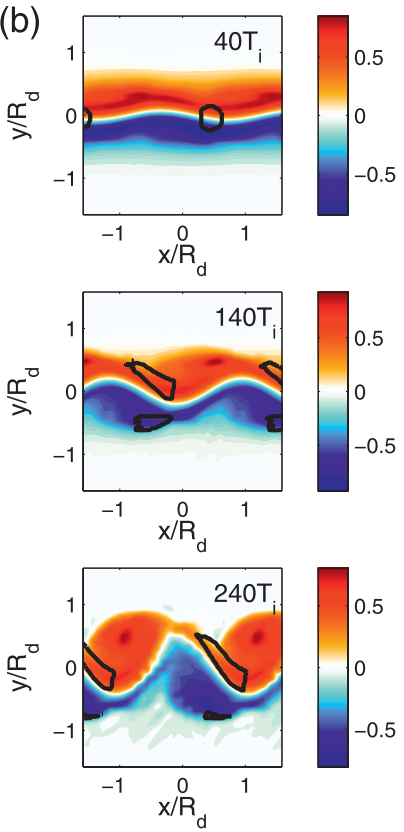
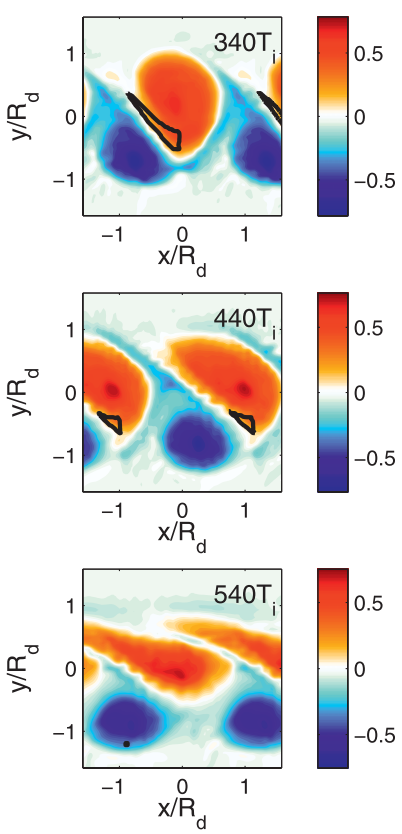

FIG. 8. Nonlinear evolution of $\zeta$ in the (a) dry and (b) moist-convective simulations. In (b), precipitation zones are situated inside the thick contours. 


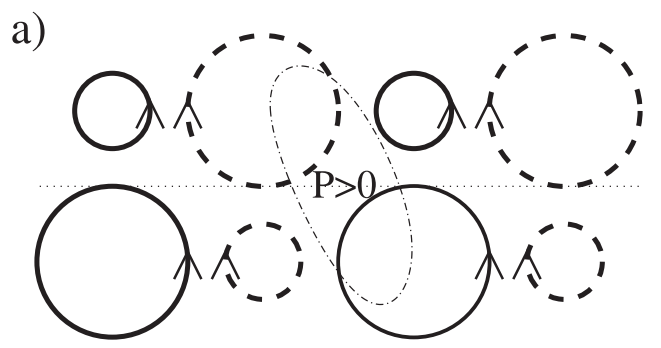

b)

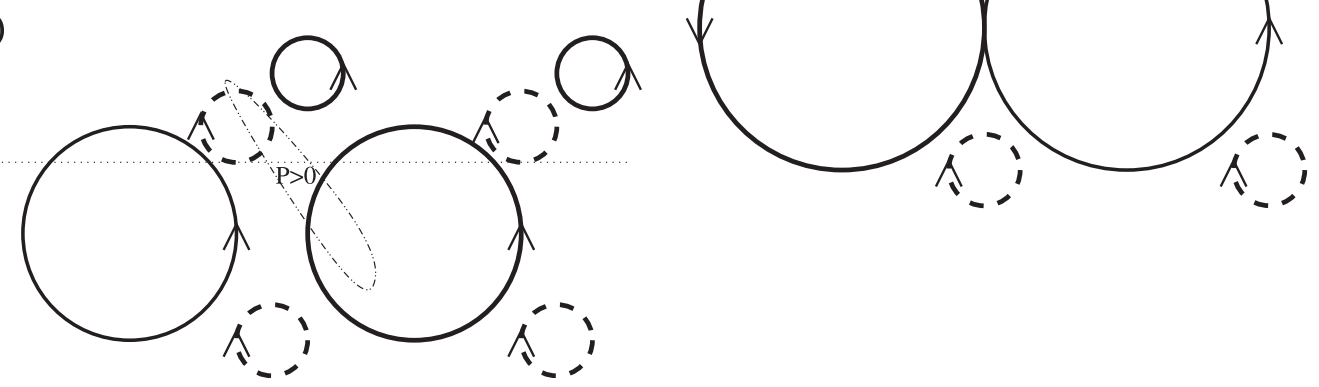

c)

FIG. 9. Sketch of the influence of precipitation on the vortex street formation as seen in Fig. 7b. Dashed and continuous circles correspond to anticyclonic and cyclonic PVCs, respectively. (a) The initial configuration; see Fig. $7 \mathrm{~b}, t=240 T_{i}$. Strong cyclonic PVCs is reinforced because of precipitation, which is indicated by the dasheddotted contour, and (b) induces a tilting effect; see Fig. 7b, panel $t=340 T_{i}$. (c) New equilibrium state after precipitation ceases; see Fig. $7 \mathrm{~b}, t=540 T_{i}$. The horizontal dotted-dashed line corresponds to $y=0$, the center line of the unperturbed jet.

anticyclonic and cyclonic vortices is clearly seen in Fig. 8b at time $t=240 T_{i}$ and later. The vortex street is progressively displaced toward the anticyclonic side of the unperturbed jet, whereas tilting of the thickness deviation (cf. Fig. 7b) corresponds to stretching of the cyclonic relative vorticity. The shift of the vortex street is clearly seen in Fig. 10, which compares the evolution of the total vorticity averaged in the $x$ direction $\langle\zeta\rangle_{x}$ in the dry and moist-convective simulations.

At later times $\left(t \gg 540 T_{i}\right.$; not shown), this stretching leads to formation of strong cyclonic and weak anticyclonic tongues at the cyclonic side of the unperturbed jet. If no more precipitation occurs, the former will merge with the cyclonic vortices of the street, whereas the latter will form an anticyclonic vorticity band.

\section{b. Fast (unbalanced) ageostrophic motions}

\section{1) DRY SIMULATION}

The ageostrophic part of the unstable mode, which was initially concentrated in its wings, is distorted during the evolution such that the IGW emission ceases to be unidirectional. This can be seen for the dry simulation in Fig. 11a describing the divergence field $\delta=\nabla \cdot \mathbf{v}$. One observes that ageostrophic motions are mostly concentrated in the slowdown areas (situated in between the strong PVCs of Fig. 7a), which are related to the strong overall relative vorticity gradients (see Fig. 8a). When the intensity of the ageostrophic motions is strong enough, they can be distinguished in $\zeta$ (see Fig. 8a for $t=240 T_{i}$ ). As expected, the intensity of these motions then decreases, because a new geostrophic equilibrium is being established.

\section{2) Moist-CONVECtive Simulation}

In the moist-convective simulation, the ageostrophic motions will trigger precipitation because $Q$ is not a Lagrangian invariant [(2.3)] and is increasing (decreasing) in the convergence (divergence) areas of the velocity field. In the quasigeostrophic approximation, the ageostrophic motions are determined by the divergence of the firstorder velocity field $\mathbf{v}^{(1)}$ and hence by the tendency of the geostrophic vorticity $\nabla^{2} \psi$,

$$
\nabla \cdot \mathbf{v}^{(1)}=-\left(\partial_{t}+\mathbf{v}^{(0)} \cdot \nabla\right) \nabla^{2} \psi .
$$

We have seen that precipitation produces ageostrophic motions, in particular in the limit of immediate relaxation $\tau \rightarrow 0$ [cf. (6.5)], so that $\nabla \cdot \mathbf{v}^{(1)}=-P / Q_{s}$ when $P>0$. This is confirmed by Fig. 11b, which displays the divergence field $\delta=\nabla \cdot \mathbf{v}$ in the moist simulation. Precipitating regions (delimited by thick contours) are indeed associated with the strong convergence areas.

Note that the ageostrophic motions directly related to precipitation (inside the precipitation zones) can be filtered by considering the effective divergence $\delta^{\star}=\nabla \cdot \mathbf{v}+$ $P / Q_{s}$. The intense ageostrophic motions can be seen in the 

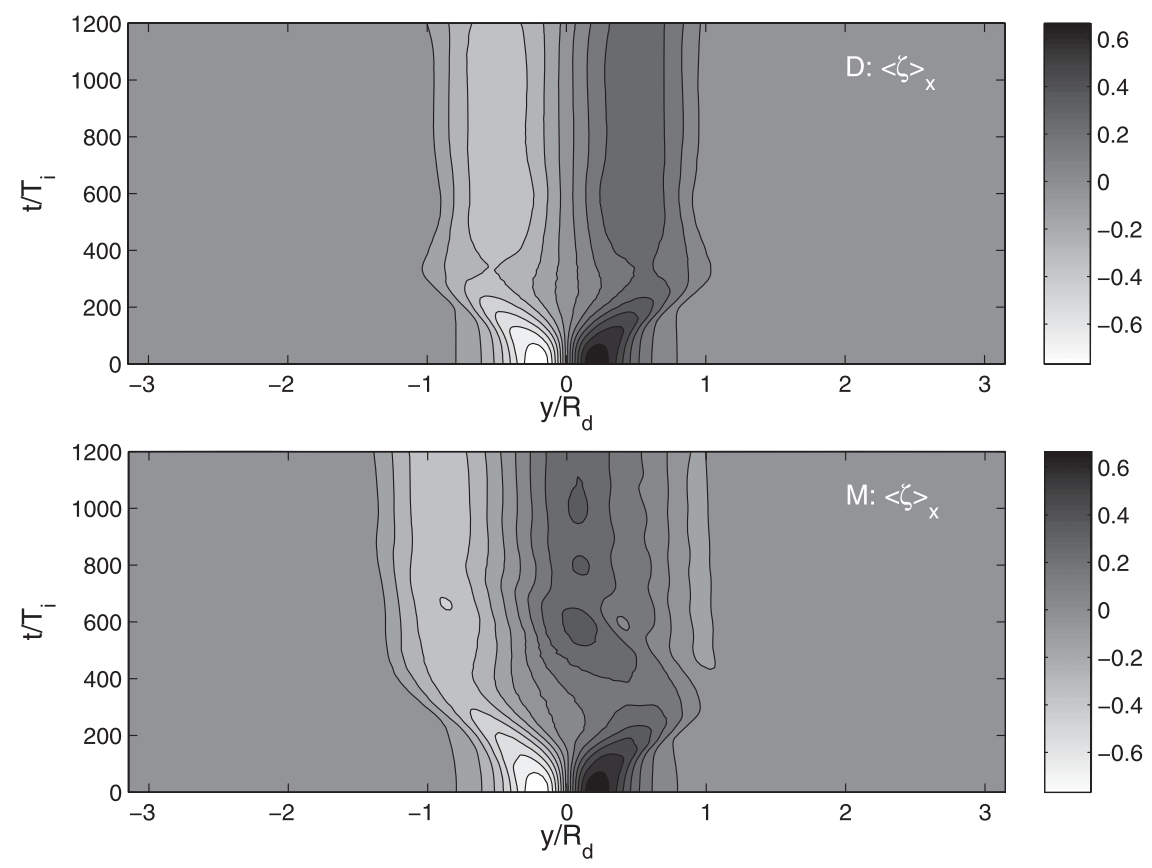

FIG. 10. Nonlinear evolution of $\langle\zeta\rangle_{x}$ in the (top) dry and (bottom) moist-convective simulations.

total relative vorticity field of Fig. 8b in a form of smallscale wiggles. They persist after the precipitation ceases (cf. Fig. $8 \mathrm{~b}$ at $t=540 T_{i}$ ) until a new geostrophic equilibrium is reached. We thus observe a "secondary" geostrophic adjustment, as compared to the dry simulation. As follows from (6.5), the precipitation-related ageostrophic motions affect the tendency of the dominant geostrophic field. This explains the prolongated influence of the moist effects on the $\eta$ field in Fig. $7 b$.

\section{c. The evolution of the moisture field}

\section{1) DRY Simulation}

The moisture can be introduced in the dry simulations presented above as a passive tracer: that is, via (2.3) with $P=0$. The anomalies of moisture created at the initial stage of the evolution (Fig. 5) are advected and mixed during the development of the instability, as shown in Fig. 12a. As for Fig. 7, the color scale varies as a function of time. Because of advection, strong gradients of moisture appear in the slowdown areas (between the dominant PVCs in Fig. 7a), and moisture anomalies are progressively caught at the borders of the vortices.

The variations of the peak value of the moisture anomaly $q^{\max }$ in the nonprecipitating simulations are presented in Fig. 13. These results allow us, on one hand, to set an appropriate background level of moisture in the simulations with precipitation and, on the other hand, to identify the influence of the velocity divergence, which makes the $q^{\max }$ change.

\section{2) Moist-CONVECTIVE Simulation}

As shown in Fig. 12b, the moisture field is first distorted as in the dry simulation, but now it does not exceed the saturation value $Q_{s}\left(q \leq q_{s}=Q_{s}-Q_{0}\right)$ because of the rapid relaxation induced by precipitation. The precipitation takes place in the positive anomalies of moisture, which are advected by the flow: some precipitation happens in the anticyclonic vortex, but it is mostly found in the zone of strong vorticity gradients (cf. Fig. $8 \mathrm{~b} ; t=240 T_{i}$ and $\left.t=340 T_{i}\right)$. The precipitation is driven by the dry convergence (cf. similar patterns of divergence in dry and moist simulations of Fig. 11).

Precipitation fronts can be observed at the interface between the precipitating area and nonprecipitating surrounding when this interface corresponds to quasi discontinuity in the moisture gradient (cf. Fig. 12b; e.g., at $x \approx-0.6 R_{d}, y=0$, and $t=340 T_{i}$ ). Their propagation has been analyzed analytically and numerically in the mcRSW system by Bouchut et al. (2009) in one spatial dimension. In two dimensions, such solutions can still be identified in the direction normal to the front.

\section{d. Comparison of the saturation of the instability in the dry and moist-convective cases}

It is clear from the preceding subsections that development and saturation of the instability in the dry and moistconvective configurations are qualitatively different. In this section, we will give quantification of this difference with the help of integral characteristics of the perturbation. 

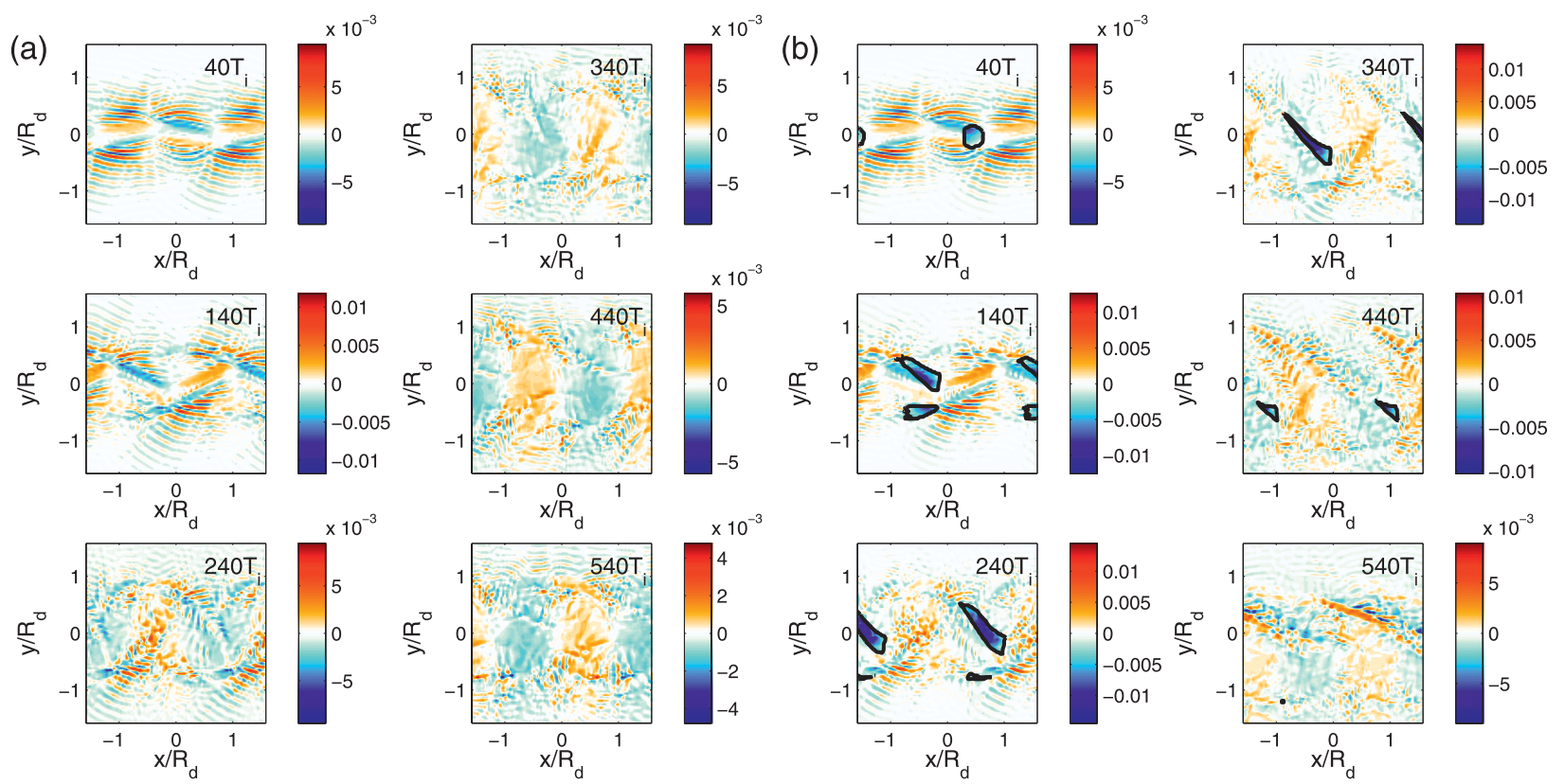

FIG. 11. Nonlinear evolution of $\delta$ in the (a) dry and (b) moist-convective simulations. In (b), the precipitation zones are situated inside the thick contours.

The evolution of the normalized dry energy norm of the perturbation $s^{2}[(5.2)]$, which was already used for estimates of the growth rates at the initial stage of the evolution in section 5, is presented in Fig. 14 (top). For the dry simulation (solid line), the norm of the perturbation grows and reaches a maximum at $t \approx 300 T_{i}$. Then, it decreases to a quasi-saturation value that corresponds to the stabilization of the vortex street $\left(t \approx 600 T_{i}\right)$. For the moist simulation (dashed line), the norm of the perturbation continues to grow and then reaches its saturation value at
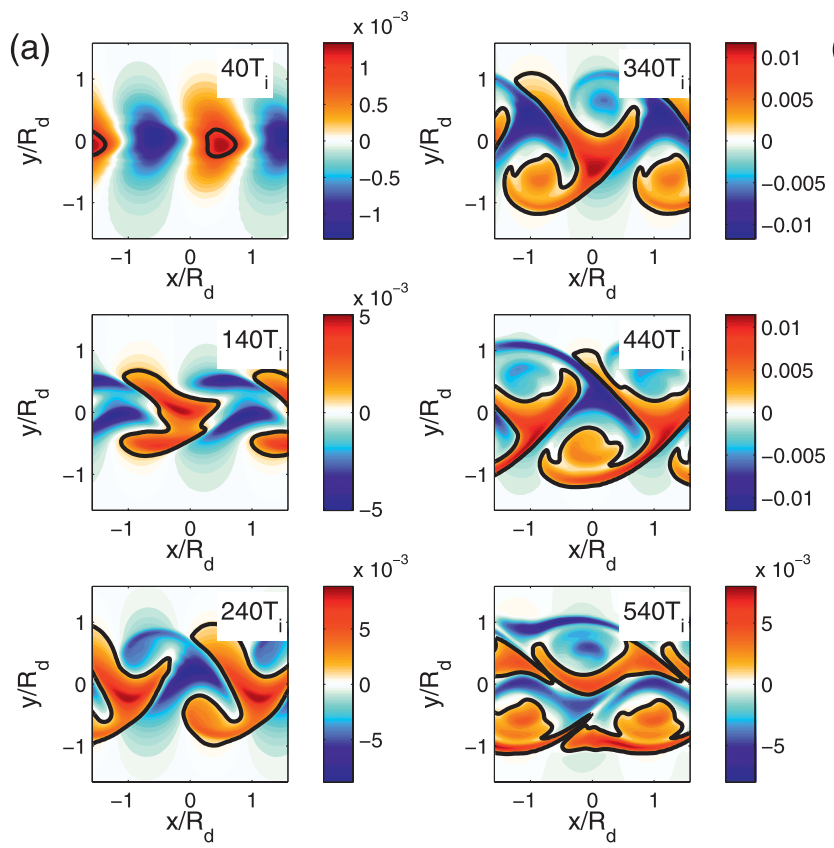
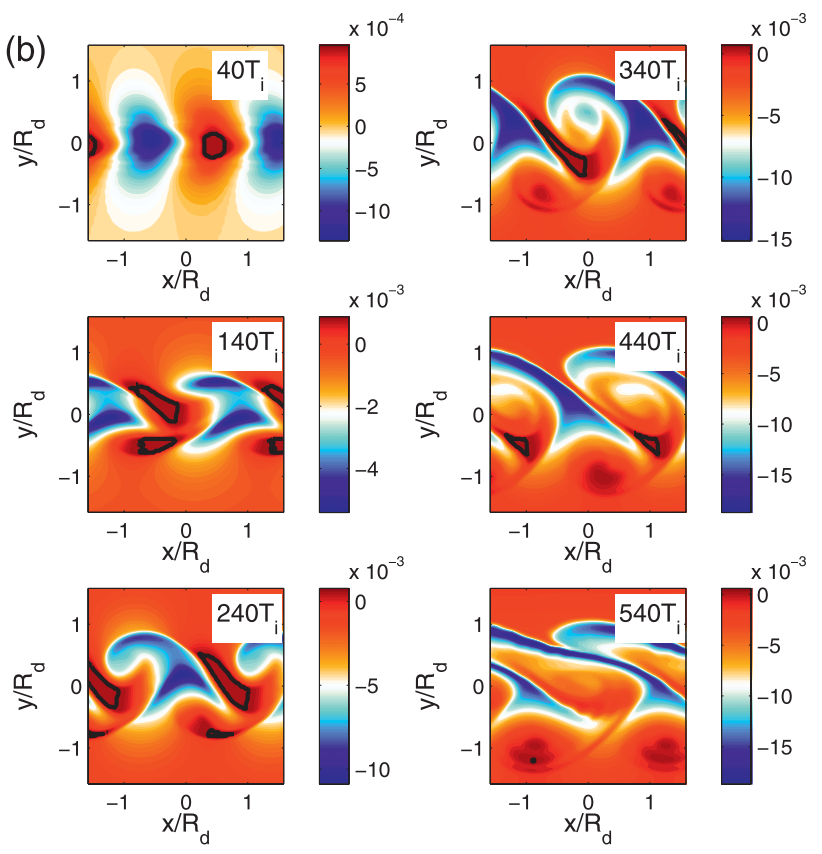

FIG. 12. Nonlinear evolution of $q=Q-Q_{0}$ in the (a) nonprecipitating and (b) moist-convective simulations. The thick line indicates the level $q_{s}=Q-Q_{0}$. Note that we change the color scale from panel to panel to better distinguish the structure of the flow. 


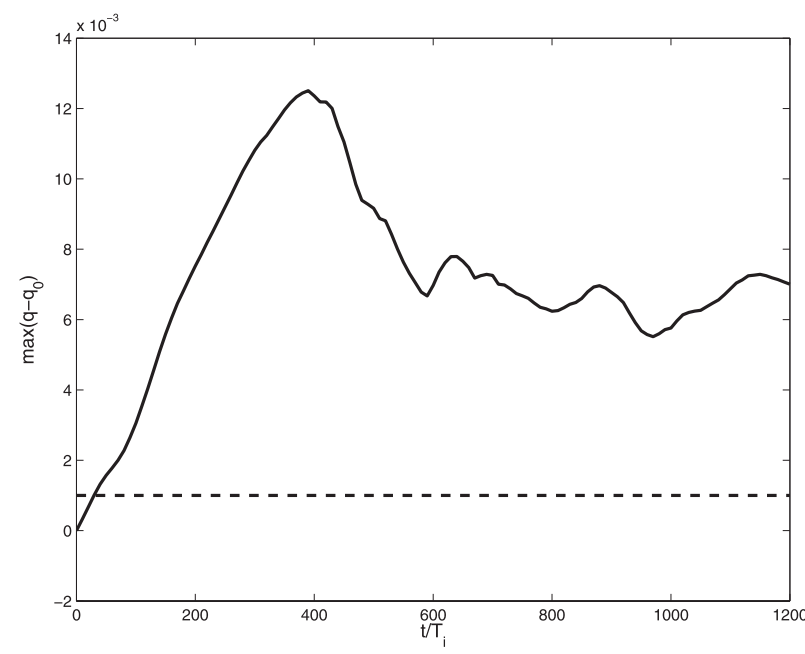

FIG. 13. Evolution of $q^{\max }=Q^{\max }-Q_{0}$ in the dry simulation with $P=0$. Also shown is the value of $Q_{s}-Q_{0}$ used in the moist simulations above (dashed line).

approximately the same time as in the dry simulation. However, this value is much larger than the one reached in the dry simulation. This is clearly related to the nonlinear moist effects described above. The separation between the dry and the moist curves becomes important at $t \approx 200 T_{i}$ when the pairs of anticyclonic PVCs are split apart by the growing strong cyclonic PVCs in the moist simulation (cf. Fig. 7b).

The behavior of the dry energy norm, particularly its potential energy part, is affected by the convective mass loss in the fluid layer, which is already mentioned in section 5 . To be sure that this effect does not substantially contaminate the diagnostics, we present in Fig. 14 (middle) the evolution of the kinetic energy norm of the perturbation, which is not directly sensitive to the mass loss. As seen from Fig. 14 (top), the enhanced efficiency of the moist-convective instability is fully confirmed in this norm. The same conclusion follows from Fig. 14 (bottom), where we plotted the evolution of the moist energy norm of the perturbation $s_{m}^{2}$, which exhibits behavior close to $s^{2}$, but with a lesser ratio of saturation values in moist and dry simulations.

The evolution of the vorticity (or enstrophy) norm of the perturbation

$$
s_{\zeta}^{t}(t)=\iint d x d y\left(\partial_{x} v-\partial_{y} u\right)^{2}
$$

is shown in Fig. 15 (top). It is associated with the quasigeostrophic motions (stronger vortices) and have behavior similar to the dry energy norm. Figure 15 (middle) represents the evolution of a norm of the ageostrophic motions for the perturbation,

$$
\rho^{2}(t)=\iint d x d y(\boldsymbol{\nabla} \cdot \mathbf{v})^{2} .
$$

It is compared to the evolution of the bulk precipitation $P_{\text {tot }}=\iint d x d y P$, which is displayed in Fig. 15 (bottom). The intensity of the ageostrophic motions is well correlated
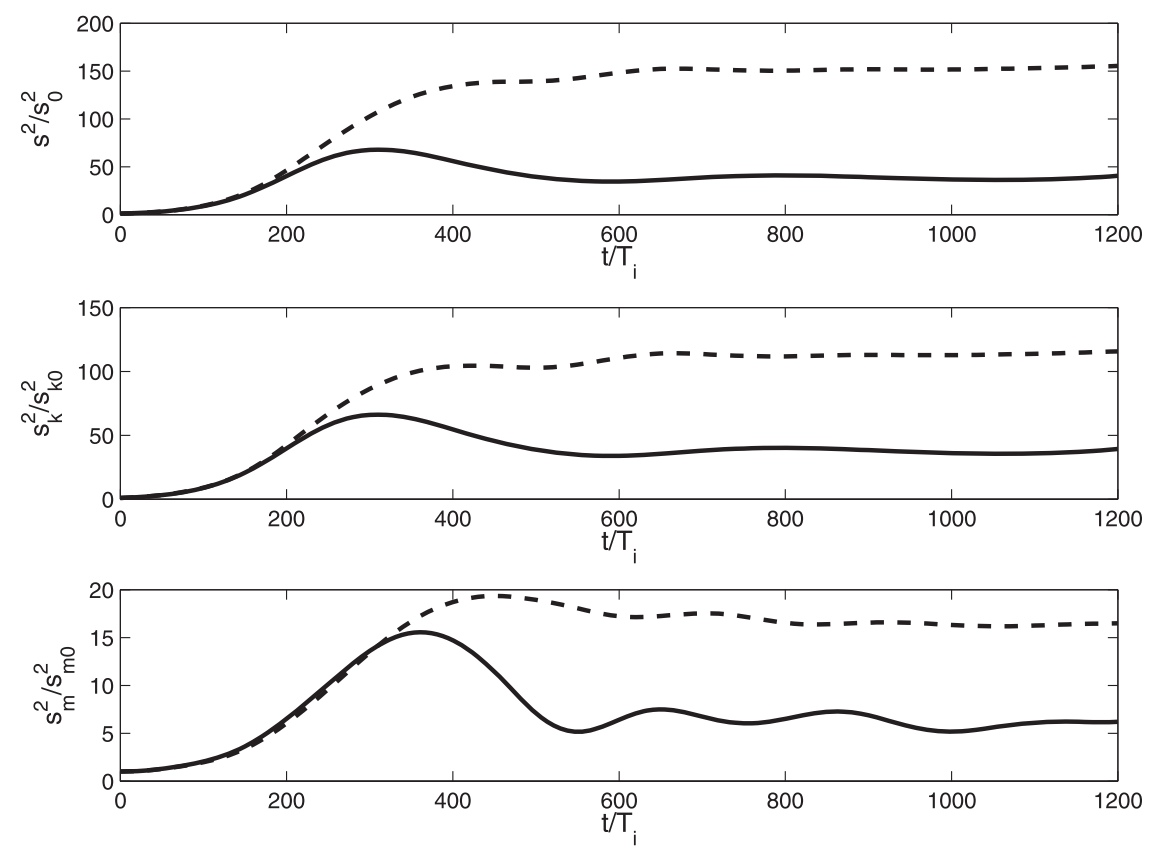

FIG. 14. Evolution of (top) $s^{2}$, (middle) $s_{k}^{2}$, and (bottom) $s_{m}^{2}$ of the perturbation in the dry (solid line) and moist (dashed line) simulations. 

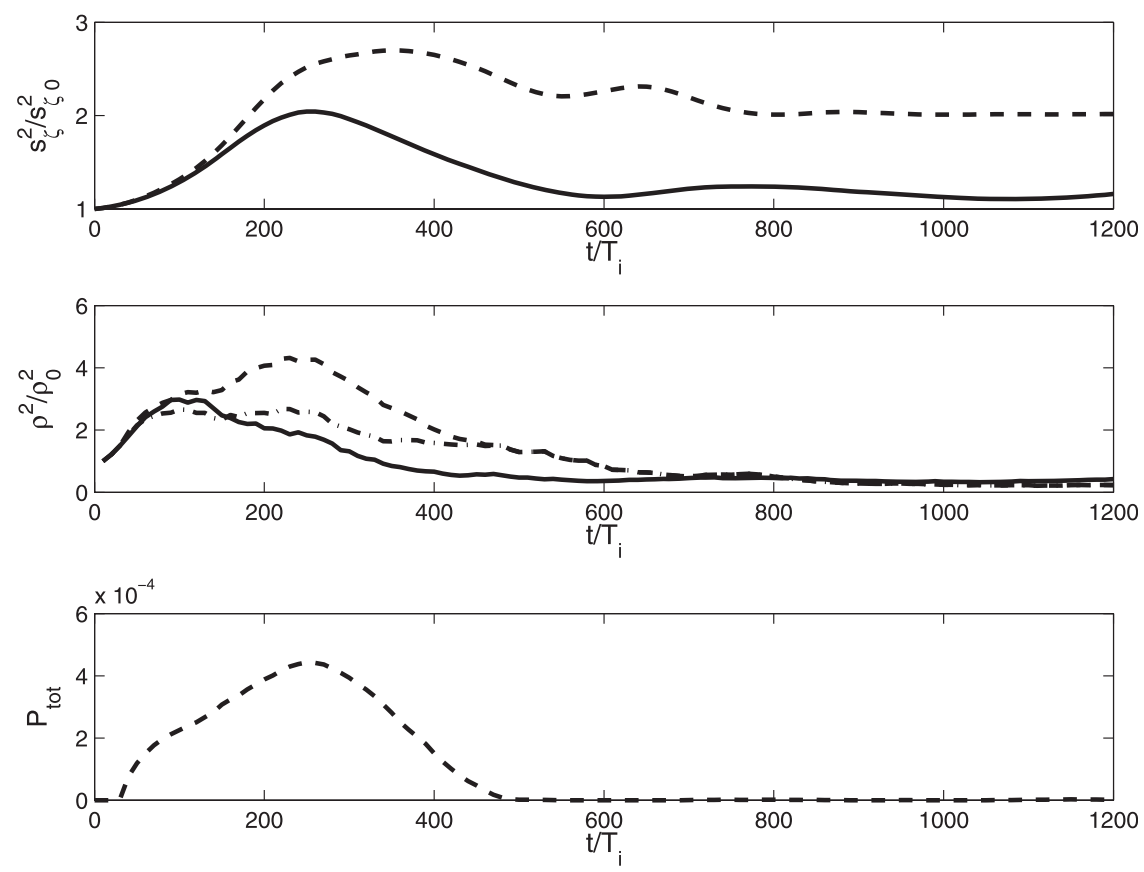

FIG. 15. Evolution of (top) $s_{\zeta}^{2}$, (middle) $\rho^{2}$, and (bottom) $P_{\text {tot }}$ in the dry (solid line) and moist (dashed line) simulations. The additional dashed-dotted line in the middle panel corresponds to the norm of ageostrophic motions based on $\delta^{\star}$ in the moist simulation.

with the precipitation and is much more pronounced in the moist simulation, in comparison with the dry one. Furthermore, the maxima of total precipitation and of $\rho^{2}$ occur about the moment of separation of the dry and moist curves $\left(t \approx 200 T_{i}\right)$ in Fig. 15 (top). The saturation of $\rho^{2}$ is clearly delayed in the moist $\left(t \approx 700 T_{i}\right)$ simulation with respect to the dry one $\left(t \approx 400 T_{i}\right)$, which means that the moist convection renders the instability more efficient. Note that the norm of the effective divergence $\delta^{\star}=\nabla \cdot \mathbf{v}+P / Q_{s}$, which appears as the dashed-dotted line in Fig. 15 (middle) allows us to verify that the ageostrophic motions are well amplified even if the direct effect of precipitation is filtered out (after $t \approx 150 T_{i}$ ). Thus the diagnostics with different norms show that the efficiency of the moist-convective instability, in the sense of the amplitude of the deviation of the end state with respect to the initial one, is substantially higher, than of the dry one.

Note that all the norms above were calculated for deviations with respect to unperturbed initial jet, according to the general scheme of nonlinear Lyapunov stability analysis. In the moist-precipitating case, they are obviously influenced by the overall mass loss due to precipitation. One could try to eliminate this effect by defining perturbations with respect to the varying zonal mean (see Fig. 16). The result is analogous to the one obtained with the previous norms until $t=400 T_{i}$ (see Figs. 14, 15). Later, however, the norms of the new perturbations saturate at lower values for the moist simulation than for the dry one.
However, a caveat concerning this analysis arises from the fact that the zonal means are different in dry and moist simulations, prohibiting a clean comparison. We see here an illustration of difficulties of quantifying predictability of the moist-convective processes. One can argue, however, that the change of zonal mean is inherent for the convective processes and should not be filtered.

Yet another way to remove the direct influence of the mass loss is to use the deviations of the perturbations with respect to the initial state from their spatial means. In this case, the evolution of the norms is similar to the results presented above (not shown).

In the context of (in)stability, an important characteristic of the zonal mean flow is its shear. A comparative calculation of the shear of the mean flow in the dry and moist simulations shows that it is getting stronger in the latter case (not presented). This observation reveals a feedback mechanism by which precipitation enhances the barotropic instability. It is, however, difficult to give an immediate explanation of this observation because of essential nonlinearity of the precipitation process.

\section{Dependence of the results on model parameters}

The results presented above depend on the choice of parameters of the model. The first group of parameters is related to the precipitation and convection parameterization: $Q_{0}, \beta$, and $\tau$. We will not discuss the dependence 

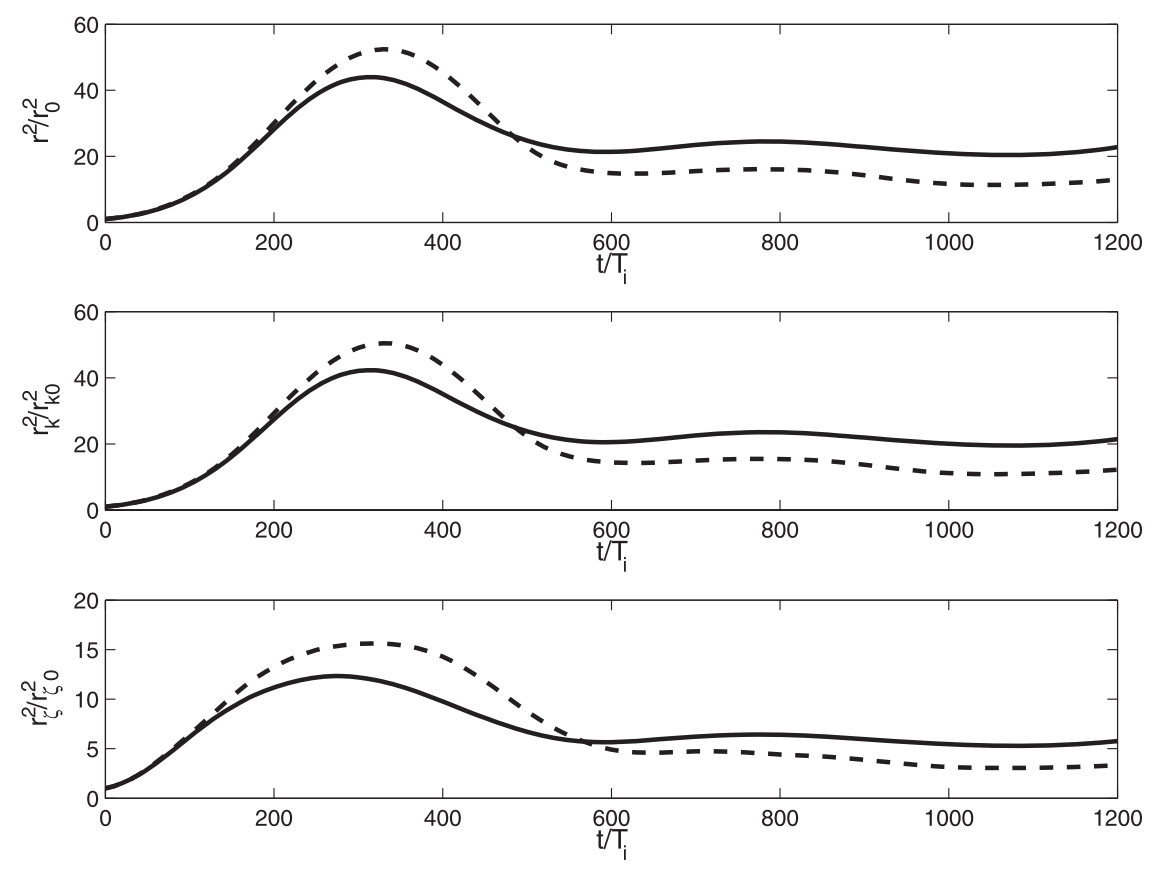

FIG. 16. Evolution of (top) $r^{2}$, (middle) $r_{k}^{2}$, and (bottom) $r_{\zeta}^{2}$ with respect to the varying zonal mean in the dry (solid line) and the moist (dashed line) simulations.

on the relaxation time $\tau$, because we have chosen it as small as possible to stay close to the quasi-equilibrium closures discussed in the introduction and section 4 .

Concerning the initial uniform background humidity field $Q_{0}$, it should be chosen in such a way as to guarantee that precipitation is indeed triggered during the instability, as was already mentioned above. Assuming $Q_{s}$ to be fixed at the value 0.9 , as discussed in section 4 , and considering the results of the dry simulation presented in Fig. 13, we see that there exists a range of values of $Q_{0}$ satisfying this condition. However, if the precipitation is triggered lately, at the end of the growth cycle of $q^{\max }$, it will not last and will not have significant dynamical influence. In such a case (e.g., for $Q_{0}=0.892$ ), we checked that precipitation is rather limited in time, and the impact of the moist mechanisms described above on the evolution of the instability is weaker.

The parameter $\beta$ governs the intensity of the influence of precipitation upon the evolution of the thickness $\eta[(2.2)]$ and upon the PV [(6.1)]. It also controls the moist characteristic velocity $c_{m}=c_{d} \sqrt{1-\left(\beta Q_{s} / H_{0}\right)}$ (cf. Bouchut et al. 2009) in precipitating regions in the limit of rapid relaxation. Consequently, to preserve the hyperbolicity of the system in these regions with such parameterization, $\beta$ should lie in the interval $0<\beta<H_{0} / Q_{s}$. For fixed parameters $Q_{s}$ and $H_{0}$, increasing the value of $\beta$ leads to slower moist velocity but to stronger moist-convection effects. For example, as shown in Fig. 17 (left) the growth rate of the perturbation after the onset of precipitation $\left(t_{P}=40 T_{i}\right)$ significantly increases with $\beta$. The fraction of the total area occupied by the precipitating regions $A_{P}$ (Fig. 17, right) strongly shrinks for high values of $\beta$, consistent with linear baroclinic results of Emanuel et al. (1987) and the nonlinear simulations in the geostrophic model of Lapeyre and Held (2004).

The second group of parameters consists of the Rossby and Burger numbers of the background jet and governs the dry instability pattern. If one changes the Rossby and/ or Burger number, both the unstable mode and the vortex street resulting from the saturation of the dry instability will be modified, as was shown by Poulin and Flierl (2003). A detailed study of essentially ageostrophic jets is beyond the scope of the present paper. Let us only mention that, because precipitation is conditioned by the ageostrophic motions, the dynamical effects of moisture will be more important for higher Rossby numbers.

Within the quasigeostrophic approximation (i.e., for low values of Ro), a lower value of $\mathrm{Bu}$ will give a more stable vortex street and will strongly reduce the transport across the jet (Poulin and Flierl 2003). In this case, we checked that vortex asymmetry produced by precipitation enhances this transport by the stretching effect (not shown). Finally, let us emphasize that the dynamical mechanisms we observe do not depend on the precise form of the jet profile, with the key feature being the existence of unstable Rossbylike mode provided by the background PV gradient. 

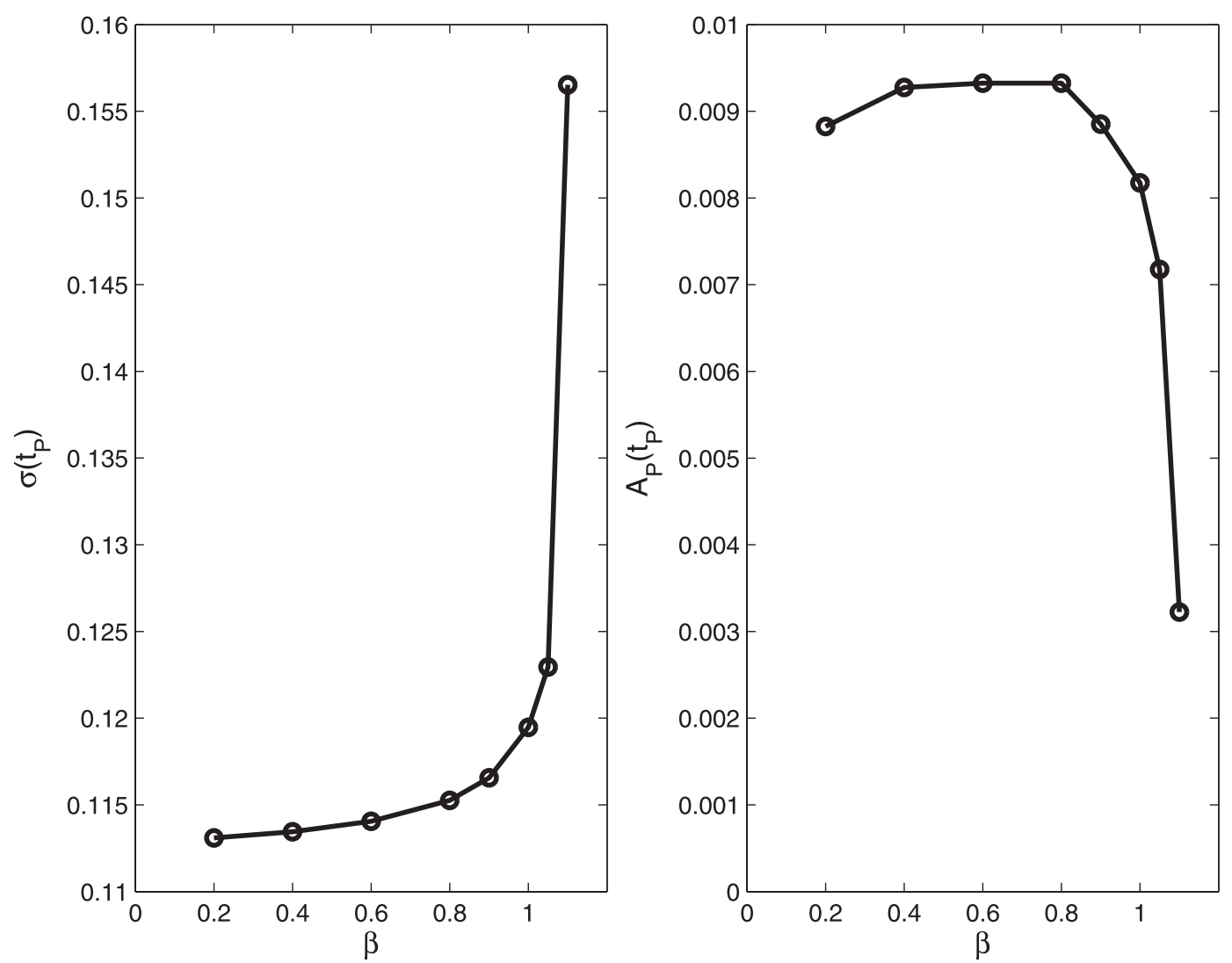

FIG. 17. The growth rate of (left) $\sigma$ and (right) $A_{P}$ after the onset of precipitation $\left(t_{P}=40 T_{i}\right)$ in the moist-convective simulation as functions of $\beta$.

\section{Summary and conclusions}

We have performed an exhaustive linear stability analysis of the Bickley jet in the rotating shallow-water model and identified the most unstable mode in a quasigeostrophic regime. It was then used to initialize numerical simulations of the nonlinear saturation of the instability with and without moist convection in the framework of the moist-convective rotating shallow-water (mcRSW) model. In this model, moist convection is parameterized through a relaxation precipitation sink, which is added into the local conservation equation for moisture. This sink is then coupled to the convective term in the mass conservation equation. The model is probably the simplest physically and mathematically consistent set of nonlinear equations, allowing us to study the dynamical effects of moisture upon the large-scale motions. We used it for a comparative study of dry and moist-convective instabilities and for a quantification of their differences. It is to be stressed that precipitation and moist-convection effects are essentially nonlinear and that traditional linear analysis of moist instabilities is impossible, which explains a general lack of knowledge on evolution of the instabilities of moist-convective systems.

Our simulations reveal substantial qualitative and quantitative differences in the development and saturation of dry and moist-convective barotropic instability. We demonstrated an overall enhancement of the efficiency of the instability in the moist-convective case, which manifests itself both in the local increase of its growth rate and in stronger departure of the end state from the initial one. The growth rate at the initial stage of development of the perturbation suddenly increases at the precipitation onset. Such behavior was observed in previous studies (e.g., Emanuel et al. 1987; Crum and Dunkerton 1992) but is established above in the presence of nonlinear precipitation threshold and without loss of hyperbolicity of the system. The deviation between the end state and the initial one is significantly larger in the moist-convective simulation, which was measured with the help of a series of perturbation norms: the dry and the moist energy norms, the kinetic energy norm, the enstrophy norm, and the norm of ageostrophic motions all giving similar results. 
We showed that positive and negative potential vorticity anomalies are affected differently by precipitation [cf. (6.1)], which results in a substantial cyclone-anticyclone asymmetry of the geostrophically balanced motions, as compared to the dry case. Consequently, the vortex street, which is the end state of the dry evolution of the instability, is deformed in the moist-convective case and tends to another balanced state. Precipitation thus indirectly causes a secondary geostrophic adjustment, which leads to deformation and displacement of the vortex street.

At the same time, moist convection significantly increases the intensity of the ageostrophic motions. As could be expected, these motions are strong in the precipitating areas, because moist convection and precipitation are directly linked to the wind convergence. However, they are also enhanced in the nonprecipitating areas, where additional unbalanced motions are generated because of the above-described secondary geostrophic adjustment.

Finally, we should emphasize that what we abusively called above an end or saturated state is, in fact, subject to a secondary modulational instability (e.g., Poulin and Flierl 2003). The role of moisture in its evolution turns to be insignificant, and we did not present in this paper the longer time simulations, which we did perform.

\section{REFERENCES}

Bennetts, D. A., and B. J. Hoskins, 1979: Conditional symmetric instability-Possible explanation for frontal rainbands. Quart. J. Roy. Meteor. Soc., 105, 945-962.

Betts, A. K., and M. J. Miller, 1986: A new convective adjustment scheme. Part II: Single columns tests using GATE wave, BOMEX, ATEX and arctic air-mass data sets. Quart. J. Roy. Meteor. Soc., 112, 693-709.

Bouchut, F., 2007: Efficient numerical finite volume schemes for shallow water models. Nonlinear Dynamics of Rotating Shallow Water: Methods and Advances, V. Zeitlin, Ed., Elsevier, 189-256.

— J. Lesommer, and V. Zeitlin, 2004: Frontal geostrophic adjustment, slow manifold, and nonlinear wave phenomena in one-dimensional rotating shallow water. Part 2. High-resolution numerical simulations. J. Fluid Mech., 514, 35-63.

,-- , and -2005 : Breaking of balanced and unbalanced equatorial waves. Chaos, 15, 013503, doi:10.1063/1.1857171.

_ _ J. Lambaerts, G. Lapeyre, and V. Zeitlin, 2009: Fronts and nonlinear waves in a simplified shallow-water model of the atmosphere with moisture and convection. Phys. Fluids, 21, 116604, doi:10.1063/1.3265970.

Charney, J. G., and A. Eliassen, 1964: On the growth of the hurricane depression. J. Atmos. Sci., 21, 68-75.

Coutinho, M. M., B. J. Hoskins, and R. Buizza, 1994: The influence of physical processes on extratropical singular vectors. J. Atmos. Sci., 61, 195-209.

Crum, F. X., and T. J. Dunkerton, 1992: analytic and numerical models of wave-CISK with conditional heating. J. Atmos. Sci., 49, 1693-1708.

Dias, J., and O. Pauluis, 2010: Impacts of convective life-time on moist geostrophic adjustment. J. Atmos. Sci., 67, 2960-2971.
Dunkerton, T. J., 1990: Eigenfrequencies and horizontal structure of divergent barotropic instability originating in tropical latitudes. J. Atmos. Sci., 47, 1288-1301.

Ehrendorfer, M., R. M. Errico, and K. D. Raeder, 1999: Singularvector perturbation growth in a primitive equation model with moist physics. J. Atmos. Sci., 56, 1627-1648.

Emanuel, K. A., Ed., 1994: Moist thermodynamic processes. Atmospheric Convection, Oxford University Press, 107-144.

—, M. Fantini, and A. J. Thorpe, 1987: Baroclinic instability in an environment of small stability to slantwise moist convection. Part I: Two-dimensional models. J. Atmos. Sci., 44, 15591573.

_ J. D. Neelin, and C. S. Bretherton, 1994: On large-scale circulations in convecting atmosphere. Quart. J. Roy. Meteor. Soc., 120, 1111-1143.

Frierson, D. M. W., A. J. Majda, and O. M. Pauluis, 2004: Large scale dynamics of precipitation fronts in the tropical atmosphere: A novel relaxation limit. Commun. Math. Sci., 2, 591-626.

Gill, A. E., 1982: Studies of moisture effects in simple atmospheric models: The stable case. Geophys. Astrophys. Fluid Dyn., 19, $119-152$.

Gula, J., and V. Zeitlin, 2010: Instabilities of buoyancy driven coastal currents and their nonlinear evolution in the two-layer rotating shallow water model. Part I. Passive lower layer. J. Fluid Mech., 659, 69-93.

Haynes, P. H., and M. E. McIntyre, 1987: On the evolution of vorticity and potential vorticity in the presence of diabatic heating and frictional or other forces. J. Atmos. Sci., 44, 828-841.

Hazel, P., 1972: Numerical studies of the stability of inviscid stratified shear flows. J. Fluid Mech., 51, 39-61.

Hoskins, B. J., and F. P. Bretherton, 1972: Atmospheric frontogenesis models: Mathematical formulation and solution. J. Atmos. Sci., 29, 11-37.

Lambaerts, J., G. Lapeyre, V. Zeitlin, and F. Bouchut, 2011: Simplified two-layer models of precipitating atmosphere and their properties. Phys. Fluids, 23, 046603, doi:10.1063/1.3582356.

Langland, R. H., R. L. Elsberry, and R. M. Errico, 1996: Adjoint sensitivity of an idealized extratropical cyclone with moist physical processes. Quart. J. Roy. Meteor. Soc., 122, 18911920.

Lapeyre, G., and I. M. Held, 2004: The role of moisture in the dynamics and energetics of turbulent baroclinic eddies. J. Atmos. Sci., 61, 1693-1710.

Lindzen, R. S., 1974: Wave-CISK in the tropics. J. Atmos. Sci., 31, 156-179.

Lipps, F. B., 1963: Stability of jets in a divergent barotropic fluid. J. Atmos. Sci., 20, 120-129.

Mak, M., 1982: On moist quasi-geostrophic baroclinic instability. J. Atmos. Sci., 39, 2028-2037.

- 1983 : On moist quasi-geostrophic barotropic instability. J. Atmos. Sci., 40, 2349-2367.

Neelin, J. D., and N. Zeng, 2000: A quasi-equilibrium tropical circulation model-Formulation. J. Atmos. Sci., 57, 17411766.

Pauluis, O., D. M. W. Frierson, and A. J. Majda, 2008: Precipitation fronts and the reflection and transmission of tropical disturbances. Quart. J. Roy. Meteor. Soc., 134, 913-930.

Philander, S. G. H., 1976: A note on the stability of the tropical easterlies. J. Meteor. Soc. Japan, 54, 328-330.

Poulin, F. J., and G. R. Flierl, 2003: The nonlinear evolution of barotropic unstable jets. J. Phys. Oceanogr., 33, 2173-2192. 
Rivière, O., G. Lapeyre, and O. Talagrand, 2009: A novel technique for nonlinear sensitivity analysis: Application to moist predictability. Quart. J. Roy. Meteor. Soc., 135, 1520-1537.

Scherer, E., and V. Zeitlin, 2008: Instability of coupled geostrophic density fronts and its nonlinear evolution. J. Fluid Mech., 613, 309-327.

Stechman, S. N., and A. J. Majda, 2006: The structure of precipitation fronts for finite relaxation time. Theor. Comput. Fluid Dyn., 20, 377-404.

Stern, M. E., 1961: The stability of thermoclinic jets. Tellus, 13, 503-508.
Trefethen, L. N., 2000: Spectral Methods in MATLAB. SIAM, $165 \mathrm{pp}$.

Wheeler, M., and G. N. Kiladis, 1999: Convectively coupled equatorial waves: Analysis of clouds and temperature in the wavenumber-frequency domain. J. Atmos. Sci., 56, 374-399.

Whitham, G. B., Ed., 1974: Water waves. Linear and Nonlinear Waves, John Wiley \& Sons, 431-484.

Zeitlin, V., 2008: Decoupling of balanced and unbalanced motions and inertia-gravity wave emission: Small versus large Rossby numbers. J. Atmos. Sci., 65, 3528-3542. 